\title{
1 Phage proteins block and trigger retron toxin/antitoxin systems
}

3

Jacob Bobonis ${ }^{1,2}$, Karin Mitosch" ${ }^{1 \#}$, André Mateus ${ }^{1 \#, ~ G e o r g e ~ K r i t i k o s ~}{ }^{1}$, Johanna R. Elfenbein ${ }^{3,4}$, Mikhail M. Savitski ${ }^{1,5,6}$, Helene Andrews-Polymenis ${ }^{7}$, and Athanasios Typas ${ }^{1,5^{*}}$

\# Equal contribution

*Correspondence: typas@embl.de

${ }^{1}$ European Molecular Biology Laboratory, Genome Biology Unit, Heidelberg, Germany

${ }^{2}$ Collaboration for joint PhD degree between EMBL and Heidelberg University, Faculty of Biosciences ${ }^{3}$ University of Wisconsin-Madison, Department of Pathobiological Sciences, Madison, WI, USA

${ }^{4}$ North Carolina State University, Department of Clinical Sciences, North Carolina, USA

${ }^{5}$ European Molecular Biology Laboratory, Structural and Computational Biology Unit, Heidelberg, Germany

${ }^{6}$ European Molecular Biology Laboratory, Proteomics Core Facility, Heidelberg, Germany

${ }^{7}$ Texas A\&M University, Department of Microbial Pathogenesis and Immunology, Texas, USA

\section{ABSTRACT}

Bacteria carry dozens of Toxin/Antitoxin systems (TAs) in their chromosomes. Upon growth, the antitoxin is co-expressed and neutralizes the toxin. TAs can be activated and inhibit growth, but when and how this occurs has largely remained enigmatic, hindering our understanding of their physiological roles. We developed TIC/TAC (Toxin Inhibition/Activation Conjugation), a high-throughput reverse genetics approach, to systematically identify molecular blockers and triggers of TAs. By applying TIC/TAC to a tripartite TA, the retron-Sen2 of Salmonella Typhimurium, we have identified multiple blockers and triggers of phage origin. We demonstrate that diverse phage functionalities are sensed by the DNA-part of the antitoxin and ultimately activate the retron toxin. Phage-origin proteins can circumvent activation by directly blocking the toxin. Some identified triggers and blockers also act on an E. coli retron-TA, Eco9. We propose that retron-TAs act as abortive-infection anti-phage defense systems, and delineate mechanistic principles by which phages trigger or block them. 


\section{INTRODUCTION}

Toxin/Antitoxin systems (TA) are prokaryotic bipartite operons consisting of an antitoxin and toxin gene pair. The antitoxin encodes a protein or an RNA, which counteracts the protein toxin. The first such system was discovered in E. coli, where $c c d B / c c d A$ "addicts" cells in stably inheriting the F-plasmid ${ }^{1}$. Plasmid-based TAs lead to addiction, because the growth of cells losing the plasmid is inhibited by the toxin, which becomes free as the antitoxin is more labile 2,3. TAs confer addictive phenotypes by additional mechanisms and/or in other mobile elements ${ }^{4,5}$. The genomics revolution unraveled that TA systems are also ubiquitous in bacterial chromosomes ${ }^{6}$. For instance, Escherichia coli K-12 encodes at least 35 chromosomal-TA systems ${ }^{7}$, whereas there are more than 80 TA systems of a specific type (type II) in the chromosome of Mycobacterium tuberculosis H37Rv 8,9. TA systems are frequently found in the accessory genomes of bacteria and change drastically between strains of the same species ${ }^{10}$. Yet, for the vast majority, we have currently little insight into what triggers them, and hence their physiological role remains elusive and intensely debated ${ }^{11}$.

A handful of TA systems have been shown to protect bacteria against phages via abortive infection (Abi) ${ }^{12-17}$. Abi is a general term describing bacterial defenses activated after a phage bypasses the bacterial innate or adaptive antiviral systems (such as Restriction-Modification ${ }^{18}$ or CRISPR-Cas systems ${ }^{19}$ ). In contrast to innate/adaptive systems which directly target the phage, Abi systems inhibit the growth of the infected bacterium, indirectly reducing phage progeny at the population-level, at the expense of the infected cell ${ }^{20}$. Although the molecular triggers of Abi systems are unknown, it is presumed that such TAs can somehow sense phage infection and activate their toxin. Interestingly, a T4-phage protein (Dmd) has been found to directly bind, and inhibit the chromosomal toxins of two such TA systems, thereby enabling the T4 phage to infect a TA-containing E. coli ${ }^{21,22}$. Thus, phages carry genes to counteract toxins of Abi TA systems (blocker genes). We reasoned that the identification of triggers or blockers of TA systems can propel our understanding of their role in bacteria.

In the accompanying manuscript, we report that the Salmonella enterica retron-Sen2 (historically named retron-ST85 ${ }^{23}$ ) encodes a novel tripartite TA system. The toxin RcaT is directly counteracted by an antitoxin unit formed by the reverse transcriptase (RT) bound to a multi-copy single-stranded DNA (msDNA) ${ }^{24}$. To elucidate its physiological role, we have developed a reverse genetics approach that enables the systematic discovery of TA blocker and trigger genes. TIC/TAC (Toxin Inhibition/Activation Conjugation) uses plasmid libraries to survey the role of all possible genome-encoded molecular cues, and takes advantage of the two-sided phenotype associated with all TAs: ectopically inducing expression of a toxin inhibits bacterial growth, while co-expressing it with its antitoxin restores growth. Using two genome- 
wide $E$. coli overexpression libraries, we identified dozens of triggers and blockers for the retron-Sen2, enriched in phage-origin proteins. Phage proteins are sensed by the retron-TA via multiple mechanisms, which are inherent to the tripartite architecture of the TA system. We propose that the retron-TA acts as an anti-phage defense system, and we provide a method that can be readily applied to uncover the physiological role of any TA system.

\section{RESULTS}

\section{A new systematic method for identifying TA blockers and triggers}

To survey for molecular cues of TA systems, we used genome-wide $E$. coli single-gene overexpression libraries (MOB; p1 ${ }^{25}$ and TransBac; p2 ${ }^{26}$ ) in tandem with strains carrying appropriate TA-expressing plasmids (Fig. 1A). We reasoned that this would allow us to identify genes that trigger and block the TA system, but are normally silent or buffered when cells are growing in lab conditions. We used a tripartite retron-TA system ${ }^{24}$ from Salmonella enterica subsp. enterica ser. Typhimurium str. 14028s (STm) as the input TA system. The retron-TA (retron-Sen2 ${ }^{27}$ ) encodes the toxin RcaT, which is inhibited directly by a complex formed between the msDNA and the reverse transcriptase (msDNA-RT) ${ }^{24}$. Overexpressing rcaT causes toxicity in E. coli, while overexpressing the entire retron-TA (msrmsd-rcaT-rrtT) alleviates the RcaT-mediated toxicity ${ }^{24}$.

To identify retron-TA triggers and blockers we developed two reverse genetics fitness-based screens called TAC (Toxin Activation Conjugation) and TIC (Toxin Inhibition Conjugation), respectively (Fig. 1A). Wildtype E. coli BW25113 carrying either an arabinose-inducible pretron plasmid (PBAD-msrmsd-rcaT-rrtT; TAC) or p-toxin plasmid (PBAD-rcaT) ${ }^{24}$ was mated with the two $E$. coli overexpression libraries (Ptac-gene- $X$ ), by high-throughput conjugation on agar plates. After selection, the resulting 18,434 double-plasmid bearing strains $(9,217$ for each screen) were grown under different induction conditions: co-inducing the retron/toxin and the library-plasmid (see TIC/TAC procedure in Methods).

We reasoned that strains that could grow upon library-plasmid or p-retron induction, but were growth-inhibited upon co-induction, carried library-plasmids containing a retron-TA trigger that activates RcaT (Fig. 1A; TAC). To identify these strains, we compared the fitness of every individual strain between the library-plasmid induction condition (IPTG; control plates) and the corresponding co-inducing conditions (Arabinose + IPTG; experiment plates) (see TIC/TAC analysis in Methods). We identified 13 triggers from the MOB library (Fig. 1B) and 10 triggers from the TransBac library (Fig. 1C) upon high trigger-induction. Fewer and slightly different triggers were identified by lower IPTG induction (ED Fig. 1A-B). Despite the high reproducibility of individual screens (ED Fig. 1C), the overlap in hits between the two libraries was low (ED 
Fig. 1D). This inconsistency has many reasons: stringent cutoffs (hence false negatives), quality of libraries (missing genes, plasmid mutations, or cloning errors), and importance of trigger levels (e.g., MOB vectors are medium-copy ${ }^{25}$, while TransBac vectors are single-copy $\left.{ }^{26}\right)$.

To ensure that the identified triggers inhibit growth by specifically activating RcaT, we selected 15 triggers, sequenced the plasmid-inserts, and conjugated them again into $E$. coli strains expressing either an empty vector, p-retron, p-retron- $\Delta r c a T$, or p-rcaT. All 15 were benign when co-expressed only with the antitoxin (p-retron- $\Delta$ rcaT plasmid), but inhibited growth when expressed with the full retron (ED Fig. 2A), suggesting that they trigger RcaT. As in the screen, different triggers displayed varying degrees of RcaT-activation, and required different IPTG induction-levels to manifest their effect (ED Fig. 2B). We noticed that several triggers, especially strong ones, were prophage-encoded genes (enrichment $p$-val $=0.01-0.025$, depending on library and induction level - Table S1) -recE (Rac-prophage), tfaP \& ymfH (e14prophage), retron RT-Eco1 (P2-like-prophage gene in E. coli BL21), and B21_03469 (prophage in E. coli BL21). Although dam is part of the E. coli core genome, Dam methylases are also commonly found in phages ${ }^{28}$. Other triggers belonged to house-keeping processes, such as translation (tufA, rp/L, rp/P, rp/V), DNA-binding/transcription ( $r d g C, r r a B$, betl, bolA), protein quality control and translocation $(\operatorname{sly} D, \sec B, y a j C)$, chorismate-tetrahydrofolate biosynthesis (folA, gcvR, aroF, aroK), but also orphan genes (ygfB, ydiE, ecpE, gnsA).

Conversely to the TAC screen, we reasoned that strains able to grow upon p-rcaT induction, carried library plasmids containing toxin blockers that inhibited RcaT, and thereby allowing for growth (Fig. 1A; TIC). We identified 9 blockers from the MOB (Fig. 1D) and 4 blockers from the TransBac library (Fig. 1E) upon low blocker induction. As for the TAC screen, data were reproducible, but most blockers were specific to library (except for $c / p A$ ) and induction conditions (ED Fig. 3). To validate these hits, we selected 11 blockers, sequenced their plasmid-inserts, and conjugated them back into $E$. coli strains expressing either a p-empty, a p-rcaT, a p-retron, or a p-retron- $\Delta$ rcaT plasmid. All blockers specifically alleviated RcaTtoxicity, while the overall fitness remained unchanged (ED Fig. 4A). Blockers inhibited RcaT to a different degree, depending on IPTG induction levels (ED Fig. 4B). Blockers were also enriched in prophage genes (enrichment $\mathrm{p}$-val $<0.01$ for MOB library and aggregated results - Table S1) - racC, ydaW (Rac-prophage), yfjH (CP4-57-prophage), yjhC (KpLE2-prophage), and $\operatorname{dicC}$ (Qin-prophage). Other blockers were related to protein quality control ( $c / p A, c / p X$, $d n a J)$, translation (infB, $d t d, t c d A$ ), TA systems (chpS), conditionally-induced pathways ( $m h p R$, $m h p E, y b e D, d s r B, g l p E, u g p C$ ) and genes affecting mating or induction levels (dapA \& ydeA). 
In summary, we developed a systematic approach to identify TA blockers and triggers (TIC/TAC), which we used to study the tripartite TA system, retron-Sen2. Many triggers and blockers had phage origins, prompting us to investigate further their underlying links to the retron.

\section{Dam triggers the retron-TA by directly methylating the antitoxin msDNA}

In E. coli, the primary DNA adenine methylase is Dam ${ }^{29}$, which methylates adenines in 5'GATC-3' DNA duplexes ${ }^{30}$. Dam was the sole retron-TA trigger that activated RcaT-toxicity even without IPTG-induction (leaky levels; ED Fig. 2). Homologues of Dam with the same methylation recognition-site are present in other bacteria (e.g., STm ${ }^{31}$ ), as well as in phages (e.g., phage $\mathrm{P} 1{ }^{32}$ ). Plasmids expressing damfrom STm, or from phage $\mathrm{P} 1$, also triggered the retron-TA (ED Fig. 5A), suggesting that the 5'-GATC-3' adenine methylation activity itself could be causing the retron-TA triggering.

Upon perturbations in msDNA biosynthesis, the endogenous RcaT in STm inhibits growth in cold-temperatures ${ }^{24}$. Overexpressing dam in STm phenocopied this, inhibiting growth in a rcaT-dependent manner at $15^{\circ} \mathrm{C}$ and $20^{\circ} \mathrm{C}$, but not at $37^{\circ} \mathrm{C}$ (Fig. $2 \mathrm{~A}$ ). This finding implied that Dam may act by directly inactivating the antitoxin unit (RT-msDNA). To test if this occurs by reducing the msDNA levels, we isolated msDNA from strains carrying a dam overexpression plasmid, or an empty vector. msDNA levels were similar in the two conditions (Fig. 2B), suggesting that dam overexpression does not affect msDNA levels.

Although msDNAs are single-stranded ${ }^{27}$, they are all reverse-transcribed from msd-RNAs containing inverted-repeats, thereby ultimately forming extended DNA hairpins, i.e., doublestranded DNA (dsDNA) ${ }^{33}$. Dam methylates adenines in 5'-GATC-3' in dsDNA. We noticed that the msDNA produced by the retron-TA contained this motif in its hairpin. To test whether msDNA methylation is involved in Dam-mediated retron-TA triggering, we mutated the Dam recognition motif on the msDNA, while retaining intact the hairpin structure (5'-GTTC-3' duplex; p-retron ${ }^{\text {mut}) . ~ W h i l e ~ c o-e x p r e s s i n g ~ d a m ~ w i t h ~ t h e ~ w i l d t y p e ~ m s D N A ~ i n h i b i t e d ~ g r o w t h ~(5 '-G A T C-3 ' ~}$ duplex; p-retron ${ }^{\mathrm{WT}}$ ), co-expressing dam with the $\mathrm{p}$-retron ${ }^{\text {mut }}$ resulted in no change in fitness (Fig. 2C). Importantly, the p-retron ${ }^{\text {mut }}$ did not affect the RcaT toxicity, since expression of $p$ retron ${ }^{\text {mut }}$ impaired growth in a $\triangle x s e A E$. coli, which produces an immature msDNA that cannot support a functional antitoxin unit ${ }^{24,34}$ (ED Fig. 5B). Furthermore, the p-retron ${ }^{\text {mut }}$ did not affect the levels or stability of msDNA (ED Fig. 5C). Overall, these results strongly suggested that Dam triggers RcaT by methylating a specific adenine of the 5'-GATC-3' duplex on msDNA. 
To prove that the msDNA did get methylated, we took advantage of the specificity of the restriction enzyme Dpnl, which cleaves only adenine-methylated 5'-GATC-3' duplexes ${ }^{35}$. We purified wildtype (5'-GATC-3') or mutated msDNA (5'-GTTC-3') from strains carrying a dam or an empty plasmid, and digested them with Dpnl. Dpnl could only cut wildtype msDNA derived from a dam overexpressing strain, but not msDNA isolated from a strain with an empty vector, nor mutated msDNA (Fig. 2D). This also suggests that the msDNA is insufficiently methylated by the endogenous Dam methylase (all strains still carry dam in chromosome), which is consistent with endogenous Dam-levels being rate limiting for higher-copy DNA elements ${ }^{36}$. Thus, Dam methylates the msDNA hairpin at the specific 5'-GATC-3' site, and triggers the retron-TA, presumably by dissociating the RT-msDNA antitoxin complex (Fig. 2E).

\section{racC-recE of Rac prophage are a linked trigger-blocker gene-pair}

Exodeoxyribonuclease VIII, encoded by recE, a Rac-prophage gene (Fig. 3A), was found to be a retron-TA trigger (Fig. 1B, ED Fig. 1-2). RecE also triggered a retron-TA from an E. coli natural isolate (retron-Eco9 24; ED Fig. 6A), suggesting that RecE interacts with a component conserved across the two retrons. RecE is known to specifically recognize double-stranded DNA, and to fully degrade one strand with a $5^{\prime} \rightarrow 3^{\prime}$ directionality ${ }^{37}$. Since msDNA from all retrons form extended hairpins (dsDNA), we wondered whether the msDNA itself is a RecEsubstrate. Indeed, we could not retrieve any msDNA from a strain overexpressing recE, while normal levels of msDNA were isolated from a strain carrying the empty-plasmid control (Fig. $3 \mathrm{~B})$. This finding suggested that RecE degrades mature msDNA in vivo, and thus reduces the functional RT-msDNA antitoxin levels.

Although overexpressing recE abolished mature msDNA production, we noticed a higher molecular-weight msDNA-band accumulating (Fig. 3B). This band was of similar molecular weight to that of immature msDNA isolated from $\triangle x s e A B$ strains ${ }^{24}$, implying that immature msDNA cannot be degraded by RecE in vivo. Indeed, overexpressing recE in a $\triangle x s e A$ strain yielded similar msDNA levels as when inducing the empty-vector control (Fig. 3C). Nevertheless, this RecE-protection only manifested in vivo, since both mature and immature msDNA were cleaved from recombinant RecE in vitro (ED Fig. 6B). Since we treated cells with RNase when isolating msDNA, this implies that the RNA-part in the immature msDNA shields the msDNA from RecE.

recE is encoded adjacently to a small prophage gene of unknown function, $\operatorname{racC}$ (Fig. 3A). RacC, a 91 amino acid protein, was the strongest retron-TA blocker identified in the TIC screen (Fig. 1D, ED Fig. 3). RacC blocked RcaT even when expressed without inducer (ED Fig. 4B), and it also blocked the toxin from retron-Eco9 (ED Fig. 6C; albeit requiring higher levels of 
induction in this case) ${ }^{24}$. Notably, overexpressing racC in STm completely blocked the RcaTmediated cold-sensitivity phenotype of all retron antitoxin deletion mutants (Fig. 3E), confirming that RacC acts directly against RcaT activity, rather than through the antitoxin.

In summary, racC-recE form a linked blocker-trigger gene-pair in the Rac prophage. RecE triggers the retron-TA by directly degrading mature msDNA, and activates RcaT (Fig. 3D), while RacC directly blocks RcaT toxicity, presumably by directly binding to RcaT (Fig. 3F) or by competitive inhibition of the RcaT target.

\section{A prophage retron-RT triggers the retron-TA by sequestering the msDNA antitoxin}

The TransBac overexpression library includes genes from the $E$. coli B strain, which are absent from the E. coli K-12 strain ${ }^{26}$. One of them, RT-Eco1 (B21_00839), is the reverse transcriptase of retron-Eco1 (retron-Ec86), which is encoded in a P2-like prophage ${ }^{38}$, and was a prominent trigger of the Sen2 retron-TA (Fig. 1C, ED Fig. 1-2). Overexpressing RT-Eco1 in STm partially activated the endogenous retron-TA, in addition to causing some cold-sensitivity on its own (Fig. 4A, ED Fig. 7). Overexpressing RT-Eco1 also triggered the retron-Eco9 (Fig. 4B), suggesting that RT-Eco1 triggers toxicity by interacting with a conserved retron component.

Since the RT-Eco1 is a retron RT, we wondered whether it disrupts the msDNA biosynthesis of the non-cognate retron-TAs. To test this idea, we isolated msDNA-Sen2 from strains carrying an RT-Eco1 or an empty plasmid. Overexpressing RT-Eco1 did not affect msDNA levels (Fig. 4C). Since the RT-msDNA interaction is essential for antitoxin activity ${ }^{24}$, we postulated that instead RT-Eco1 could be competing with RT-Sen2, for binding to the mature msDNA-Sen2. This competition would free RT-Sen2 from its cognate msDNA, rendering the antitoxin unit inactive, and therefore activate RcaT. In this case supplying extra copies of msDNA, while maintaining constant protein-levels of the two RTs, would reduce the competition for msDNA-binding, and alleviate the RT-Eco1-mediated triggering of RcaT.

The limiting step in msDNA synthesis are the msrmsd-RNA template-levels, not the RT protein-levels, which produce more msDNA if given more msrmsd-RNA substrate. To supply more msDNA, we overexpressed only the msrmsd-RNA template from a third plasmid. To exclude that RT-Eco1 interacts with the msrmsd-RNA itself, we also supplied msrmsdmut template, which cannot be reverse transcribed into msDNA (due to a mutation in the branching $\mathrm{G}$ of the $m s r$ region ${ }^{39}$ ). Indeed, supplementing msrmsd $^{\mathrm{WT}}$, but not msrmsd ${ }^{\text {mut }}$, completely abolished RT-Eco1 triggering (Fig. 4D). At high IPTG induction-levels, msrmsd ${ }^{\text {mut }}$ triggered the retron-TA by itself, presumably because msrmsdmut competes for binding with the native 
251 msrmsd template (Fig. 4D). In all cases, toxicity was due to RcaT, since none of the constructs

252 inhibited growth upon co-expressing a p-retron- $\Delta$ rcaT control vector (Fig. 4D).

253

254 Thus, the RT-Eco1 acts as a retron-TA trigger, by competing with the RT-Sen2 and

255 sequestering its non-cognate msDNA-Sen2 from the RT-msDNA antitoxin complex. This 256 activates the toxin RcaT (Fig. 4E). 


\section{DISCUSSION}

We have developed a reverse genetics-based method, TIC/TAC, which uses systematic gene overexpression libraries to identify molecular blockers and triggers of TA systems. Overexpression libraries allow us to query the role of genes, which are normally not expressed or kept under tight control in standard laboratory growth conditions, when the TA remains inactive. Such genes are more likely to serve as molecular cues for the TA system. One of the requirements for TIC/TAC is for the studied TA system to work in E. coli, or in phylogenetically related enterobacteria, where F-based plasmid conjugation of the overexpression libraries works. The functionality of TA systems from diverse phyla has been routinely assessed in $E$. coli $13,14,17,40$, hence TIC/TAC can be readily applied to many TA systems. As more overexpression libraries become available, more endogenous molecular cues can be probed. Alternatively, the use of knockdown or knockout libraries ${ }^{41-44}$ can also provide insights into triggers or blockers, albeit more indirectly. An advantage of using $E$. coli overexpression libraries for TIC/TAC is the unrivaled functional characterization of the E. coligenome ${ }^{45}$, which facilitates mechanistic studies on potential hits.

We applied TIC/TAC to a new tripartite TA encoded by bacterial retron elements: the toxin RcaT is inhibited by protein-protein interactions with an RT-msDNA complex ${ }^{24}$. We identified multiple triggers and blockers, the majority of which we could validate in targeted assays, confirming that TIC/TAC has few false positives. Some of the identified hits likely point to the target and function of RcaT and/or may feed into the complex biosynthesis of the antitoxin complex ${ }^{24}$. Among the hits, we noticed that many were phage-related triggers (dam, recE, RTEco1, B21_00839, ymfH, tfaP) and prophage-gene blockers ( $r a c C, \operatorname{dicC}, y d a W, y f j H, y j h C)$, suggesting an extensive arms-race between the retron-TA and phages. In agreement with our findings, an independent study showed that retrons act as bacterial abortive infection antiphage defense systems ${ }^{46}$. We provide insights into the underlying mechanisms through which retron-TAs sense phage attack. In all tested cases, it is the inherent architecture of the tripartite retron-TA systems, and the presence of a DNA component in the antitoxin (msDNA) that enables retrons to sense phages - coupling the sensing to functions needed for phage proliferation.

It is important to note that triggers such as Dam and RecE are also simultaneously antirestriction genes, used by phages to defend against type II ${ }^{47}$ or type III ${ }^{48}$ restrictionmodification systems (RM), respectively. Thus, phage defenses against innate bacterialdefense systems (anti-RM proteins), can inadvertently trigger retron-TAs by directly inactivating the RT-msDNA antitoxins, leading to RcaT-mediated abortive infection (Fig. 5). It still remains to be understood why the toxin is active only at cold or anaerobic conditions ${ }^{24}$ 
and whether this relates to the specific lifestyle of STm (e.g., the environment has ambient temperatures and gut is anaerobic), or whether phage infection provides additional cues for RcaT to become active in aerobic conditions at $37^{\circ} \mathrm{C}$. Nevertheless, our study suggests the presence of crosstalk between abortive infection systems (Abis) and innate/adaptive antiphage defenses (RM, CRISPR/Cas), where Abis are directly triggered by phage-products meant to block RM or CRISPR/Cas systems (Fig. 5) ${ }^{49}$.

How chromosomal-TAs are triggered has been a heavily debated question. The idea that chromosomal-TAs are triggered similarly to plasmid-based TAs, where labile antitoxins are preferentially degraded by stress-induced bacterial proteases, has dominated the field for a long time ${ }^{14,50-55}$. Yet, chromosomal-TAs do not confer addictive phenotypes to mobile DNA elements ${ }^{56-58}$, implying that their antitoxins are not labile ${ }^{59}$. In contrast to non-bound antitoxins, toxin-bound antitoxins are often resistant to protease degradation, and maintain their toxins inactive even under protease-stimulating conditions ${ }^{58}$. These findings together with the fact that key reports on links between stress, proteases, and TA activation were later discovered to be confounded by biological artefacts ${ }^{54,55,60,61}$ has reopened the discussion on how chromosomal-TA systems could be activated. Our study provides evidence into how TA systems can be activated by phages. We demonstrate that triggers can directly inactivate the RT-msDNA antitoxin by methylating, degrading, or simply just binding to its msDNA component. This concept is in stark contrast with models where the cue is part of a general stress response (e.g., bacterial proteases or phage-induced host-alterations ${ }^{12,62}$ ), which would indirectly induce multiple TA systems. We postulate that more TA systems will have direct and specific cues that interfere with antitoxin or toxin activity, which our TIC/TAC methodology could help to identify. This in turn will propel our understanding of the myriad TA systems found in prokaryotes.

The reasons for multiple TA systems existing in bacterial genomes have also been heavily debated ${ }^{63}$. In the case of the retron-TA, the trigger-specificity is narrow. For example, Dammediated triggering requires that the targeted msDNA contains the 5'-GATC-3' recognition site in its dsDNA-hairpin sequence. Additionally, msDNA not cleaved by exonuclease VII (immature msDNA) is immune to recE-mediated degradation. For most retrons, exonuclease VII is not involved in msDNA biosynthesis ${ }^{33}$. This finding implies that TA systems (even of the same type) have different triggers, thereby providing one possible explanation for the requirement for high numbers of predicted TAs per genome.

In summary, retron-TAs are tripartite abortive-infection systems. The presence of the hairpin DNA component in the antitoxin enables direct sensing of different facets of essential phage 
activities. Retrons express thousands of copies of linear ssDNA-hairpins intracellularly (msDNA) ${ }^{64}$, and encode the antitoxin activity in the RT-msDNA complex ${ }^{24}$. Applying the TIC/TAC screen developed here to more Abi and TA systems will not only expand our understanding of these fascinating modules, but will also offer new paths for specifically treating bacterial pathogens, either through directly triggering endogenous TAs, or through empowering phage therapy to identify phages that are resistant to such defense systems.

\section{ACKNOWLEDGEMENTS}

We thank members of the Typas lab for discussions, and especially Morgane Wartel for assistance in establishing the high-throughput conjugation protocol. This work was supported by the European Molecular Biology Laboratory and the Sofja Kovaleskaja Award of the Alexander von Humboldt Foundation. AM and KM were supported by a fellowship from the EMBL Interdisciplinary Postdoc (EI3POD) programme under Marie Skłodowska-Curie Actions COFUND (grant number 664726). JRE was supported by the National Institutes of Health (K08Al108794). HAP is supported by NIFA (NIFA 2016-11004 \& 2017-08881) and DARPA. AT is supported by an ERC consolidator grant, uCARE.

\section{AUTHOR CONTRIBUTIONS}

JRE, MMS, HAP, \& AT supervised the study. JB and AT conceived the study. JB, KM, AM \& AT designed the experiments. JB, KM \& AM performed them. GK analyzed the TIC/TAC screen data. JB, AM \& GK designed figures, with inputs from AT. JB \& AT wrote the manuscript, with input from all authors.

\section{DATA AVAILABILITY STATEMENT}

All raw/processed data from TIC/TAC screens can be found in Table S2. All unprocessed source images are available upon request.

\section{CODE AVAILABILITY STATEMENT}

The code used for TIC/TAC analysis is available upon request.

\section{COMPETING INTEREST DECLARATION}

We declare no competing financial interests.

\section{ADDITIONAL INFORMATION}

Supplementary information is available for this paper. Correspondence and requests for materials should be addressed to AT (typas@embl.de). 


\section{FIGURE LEGENDS}

369 Figure 1. Toxin Inhibition/Activation Conjugation (TIC/TAC): a high-throughput reverse genetics approach to discover TA blockers and triggers.

(A) Two systematic, arrayed, conjugative-proficient E. coligene overexpression libraries (Ptacgene 1 to Ptac-gene X; library-plasmids ${ }^{25,26}$ ) are transferred into recipient strains of the desired

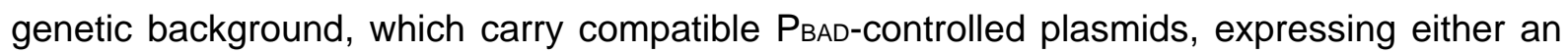
antitoxin-toxin (PBAD-TA), or a toxin (PBAD-T). Inducing the $\mathrm{P}_{B A D}-T$ plasmid inhibits growth, while inducing the PBAD-TA plasmid does not. Co-inducing library-plasmids carrying a TA-trigger results into TA-mediated growth inhibition (TAC), while co-inducing library-plasmids carrying a TA-blocker alleviates toxin-mediated growth inhibition (TIC).

(B-C) TAC screen using the IPTG-inducible library-plasmids from the MOB (B; p1) ${ }^{25}$ and TransBac (C; p2) ${ }^{26}$ libraries, respectively. Arrayed libraries (384-density format) were conjugated in an $E$. coli recipient carrying an arabinose-inducible p-retron plasmid. Transconjugants carrying both plasmids were selected by antibiotics and auxotrophies (see Methods), individual colony integral opacities were measured ${ }^{65}$, and z-scores of strains were calculated after growing on experiment plates containing arabinose + low $(0.1 \mathrm{mM})$ or high (1 mM) IPTG, or on control plates containing only low or high IPTG. Z-score differences per strain (y-axes) were derived by subtracting z-scores in control plates, from z-scores in experiment plates ( $n=2$; see Table S2 for scores). Scores shown from high-IPTG induction (see ED Fig. 1A-B for low-IPTG results). Chromosomal position (x-axes) is according to the gene start and E. coli MG1655 coordinates. Grey line denotes the hit cut-off (Z-score difference $>1$ ), and phage-related trigger-genes are in bold.

(D-E) TIC screen using the IPTG-inducible library-plasmids from the MOB (D; p1) ${ }^{25}$ and E. coli recipient carrying an arabinose-inducible p-rcaT plasmid. Axes and data analysis as described in B-C, but Z-score differences were derived by subtracting z-scores of experiment plates from control plates. Scores shown from low-IPTG induction (see ED Fig. 3A for MOB high-IPTG results). Grey line denotes the hit cut-off (Z-score difference $>4$ ), and phage-related blocker-genes are in bold.

Figure 2. Dam triggers the retron-TA by directly methylating msDNA.

(A) Overexpressing Dam triggers the endogenous STm retron-TA. STm strains (WT, $\Delta$ rcaT) carrying plasmid p1-dam or an empty vector ( $\mathrm{p} 1$-empty) were grown for 5 hours in ampicillinLB, serially diluted, spotted on ampicillin-LB plates containing IPTG (low), and then incubated at $15^{\circ} \mathrm{C}, 20^{\circ} \mathrm{C}$, or $37^{\circ} \mathrm{C}$. Representative data shown from two independent experiments. 
were co-induced with arabinose and IPTG (low). Extracted msDNA was electrophoresed in a TBE-Polyacrylamide gel. A representative gel of three independent experiments is shown.

(C) Mutating the 5'-GATC-3' motif in the msDNA duplex abolishes Dam-triggering, without affecting duplex formation. E. coli BW25113 carrying combinations of plasmids p-retron ${ }^{W T}$, pretron $^{\text {mut }}$ (5'-GTTC-3' mutation), p1-dam, and empty vectors (p-empty, p1-empty) were grown for 5 hours in LB with appropriate antibiotics, serially diluted, spotted on LB plates containing antibiotics, IPTG (low), and arabinose, and incubated at $37^{\circ} \mathrm{C}$. Representative data shown from two independent experiments.

(D) Dam methylates the 5'-GATC-3' site on msDNA. msDNA was isolated from STm strains carrying plasmids $p$-retron ${ }^{\mathrm{WT}}$ or $p$-retron ${ }^{\mathrm{mut}}$, and $\mathrm{msDNA}^{\mathrm{WT}} / \mathrm{msDNA}^{\text {mut }}$ were purified further by gel-extraction. Purified msDNA was digested with Dpnl, which recognizes and cuts methylated 5'-GATC-3' sites, and digests were electrophoresed on a denaturing TBE-polyacrylamide gel (msDNA runs higher in denaturing compared to native polyacrylamide gels).

(E) Dam triggers the retron-TA by methylating the msDNA. This results into an active RcaT (active toxin in black) - here shown to occur by dissociation of the msDNA from the RT (hypothetical).

\section{Figure 3. Rac prophage genes racC-recE are a blocker-trigger gene-pair.}

(A) racC and recE are linked Rac-prophage E. coli genes. The Rac prophage in E. coli BW25113 contains recE (exodeoxyribonuclease VIII), preceded by a small orphan gene, racC.

(B) RecE degrades mature msDNA in vivo. msDNA was extracted from STm strains carrying combinations of plasmids $\mathrm{p}$-retron- $\Delta r c a T$ with $\mathrm{p} 1-r e c E$, or $\mathrm{p} 1$-empty, respectively. Plasmids were co-induced with arabinose and IPTG (low). Extracted msDNA were electrophoresed in a TBE-Polyacrylamide gel. Mature msDNA runs close to the 50bp ladder band (double stranded DNA), whereas unprocessed DNA runs slightly higher and accumulates upon RecE overexpression. Representative results from three independent experiments are shown.

(C) Immature msDNA cannot be degraded by RecE in vivo. Same as in panel B, but msDNA were isolated from STm $\Delta x s e A$ strains. Representative results from two independent experiments are shown.

(D) Model of RecE triggering the retron-TA by degrading msDNA. RecE directly degrades the mature msDNA, resulting in dissociation of the RT-msDNA complex, and activation of RcaT.

(E) RacC blocks cold-sensitivity of endogenous STm retron-antitoxin deletions. STm strains (WT, $\Delta r r t T, \Delta x s e A, \Delta r n h A, \Delta m s r m s d$ ) carrying plasmid p1-racC or an empty vector (p1-empty) were grown for 5 hours in ampicillin-LB, serially diluted, spotted on ampicillin-LB plates containing IPTG (low), and incubated at $15^{\circ} \mathrm{C}$, or $37^{\circ} \mathrm{C}$. Representative data shown from two independent experiments. 
(F) Model of RacC blocking the retron-TA by inhibiting RcaT. RacC inhibits the toxic activity of RcaT, presumably by directly binding to RcaT.

Figure 4. RT-Eco1 triggers the retron by sequestering the msDNA-Sen2 from RT-Sen2.

(A) Overexpressing RT-Eco1 partially triggers the endogenous STm retron-TA. STm strains hours in tetracycline-LB, serially diluted, spotted on tetracycline-LB plates containing IPTG (low), and incubated at $15^{\circ} \mathrm{C}, 20^{\circ} \mathrm{C}$, or $37^{\circ} \mathrm{C}$. Representative data shown from two independent experiments.

450 (B) RT-Eco1 triggers the retron-Eco9 from E. coli NILS-16 ${ }^{24}$. E. coli BW25113 carrying combinations of plasmids p-retron-Eco9, p-retron-ArcaT-Eco9, p2-RT-Eco1, and p2-empty, were grown for 5 hours in LB with appropriate antibiotics, serially diluted, spotted on LB plates with antibiotics, arabinose, and IPTG (high), and incubated at $37^{\circ} \mathrm{C}$. Representative data shown from three independent experiments.

(C) RT-Eco1 does not affect msDNA levels. msDNA was extracted from STm $\Delta$ retron cells being complemented by $p$-retron- $\Delta r c a T$, and carrying p2-RT-Eco1 or p2-empty. Plasmids were co-induced with arabinose and IPTG (high). Extracted msDNAs were electrophoresed in a TBE-Polyacrylamide gel, and a representative gel from two independent experiments is shown.

460 (D) msDNA overexpression alleviates RT-Eco1 triggering. E. coli BW25113 carrying combinations of plasmids p-retron, p-retron- $\Delta r c a T$, p1-msrmsd ${ }^{\mathrm{WT}}, \mathrm{p} 1$-msrmsd ${ }^{\text {mut }}$, p2-RT-Eco1, and empty vectors (p1-empty, p2-empty) were grown and spotted as in B, but in different antibiotics and IPTG concentrations.

(E) RT-Eco1 triggers the retron-TA by sequestering msDNA away from its native RT. RT-Eco1 titrates msDNA-Sen2, resulting in unloaded RT-Sen2, which cannot alone neutralize the toxicity of RcaT.

Figure 5. Retrons are Abi systems protecting against phage defense. Phages encode anti-restriction proteins (green-blue circle) to counteract bacterial Restriction-Modification systems (early-defense systems; RM). These anti-RM proteins (Dam, RecE) trigger the retronTA by directly interacting with the msDNA, which disrupts the antitoxin. The latter allows the toxin (RcaT) to inhibit the growth of the infected bacterium, and thereby to indirectly stop the phage propagation - abortive infection (Abi). Thus, phage proteins are simultaneously blockers for bacterial innate defenses and triggers of secondary Abi defense systems (hence green-blue color). Being an arms race, phages have also evolved blocker proteins (blue), able to directly inhibit toxin activity of triggered Abi systems (e.g., RacC). 
bioRxiv preprint doi: https://doi org/10.1101/2020.06.22.160242; this version posted June 22, 2020. The copyright holder for this preprint (which was not certified by peer review) is the author/funder, who has granted bioRxiv a license to display the preprint in perpetuity. It is made available under aCC-BY 4.0 International license.

\section{REFERENCES}

1. Ogura, T. \& Hiraga, S. Mini-F plasmid genes that couple host cell division to plasmid proliferation. Proc. Natl. Acad. Sci. U. S. A. 80, 4784-4788 (1983).

2. Van Melderen, L., Bernard, P. \& Couturier, M. Lon-dependent proteolysis of CcdA is the key control for activation of CcdB in plasmid-free segregant bacteria. Mol. Microbiol. 11, 1151-1157 (1994).

3. Lehnherr, H. \& Yarmolinsky, M. B. Addiction protein phd of plasmid prophage P1 is a substrate of the ClpXP serine protease of Escherichia coli. Proc. Natl. Acad. Sci. U. S. A. 92, 3274-3277 (1995).

4. Gerdes, K., Rasmussen, P. B. \& Molin, S. Unique type of plasmid maintenance function: Postsegregational killing of plasmid-free cells. Proc. Natl. Acad. Sci. U. S. A. 83, 3116-3120 (1986).

5. Lehnherr, H., Maguin, E., Jafri, S. \& Yarmolinsky, M. B. Plasmid addiction genes of bacteriophage P1: doc, which causes cell death on curing of prophage, and phd, which prevents host death when prophage is retained. Journal of Molecular Biology 233, 414-428 (1993).

6. Pandey, D. P. \& Gerdes, K. Toxin-antitoxin loci are highly abundant in free-living but lost from hostassociated prokaryotes. Nucleic Acids Res. 33, 966-976 (2005).

7. Harms, A., Brodersen, D. E., Mitarai, N. \& Gerdes, K. Toxins, Targets, and Triggers: An Overview of Toxin-Antitoxin Biology. Mol. Cell 70, 768-784 (2018).

8. Ramage, H. R., Connolly, L. E. \& Cox, J. S. Comprehensive Functional Analysis of Mycobacterium tuberculosis Toxin-Antitoxin Systems: Implications for Pathogenesis, Stress Responses, and Evolution. PLoS Genet. 5, e1000767 (2009).

9. Shao, Y. et al. TADB: a web-based resource for Type 2 toxin-antitoxin loci in bacteria and archaea. Nucleic Acids Res. 39, D606-D611 (2011).

10. Horesh, G. et al. Type II and type IV toxin-antitoxin systems show different evolutionary patterns in the global Klebsiella pneumoniae population. Nucleic Acids Res. 48, 4357-4370 (2020).

11. Fraikin, N., Goormaghtigh, F. \& Van Melderen, L. Type II Toxin-Antitoxin Systems: Evolution and Revolutions. J. Bacteriol. 202, (2020).

12. Pecota, D. C. \& Wood, T. K. Exclusion of T4 phage by the hok/sok killer locus from plasmid R1. J. Bacteriol. 178, 2044-2050 (1996).

13. Blower, T. R. et al. Identification and classification of bacterial Type III toxin-antitoxin systems encoded in chromosomal and plasmid genomes. Nucleic Acids Res. 40, 6158-6173 (2012).

14. Sberro, H. et al. Discovery of Functional Toxin/Antitoxin Systems in Bacteria by Shotgun Cloning. Mol. Cell 50, 136-148 (2013).

15. Dy, R. L., Przybilski, R., Semeijn, K., Salmond, G. P. C. \& Fineran, P. C. A widespread bacteriophage abortive infection system functions through a Type IV toxin-antitoxin mechanism. Nucleic Acids Res. 42, 4590-4605 (2014).

16. Dedrick, R. M. et al. Prophage-mediated defence against viral attack and viral counter-defence. Nat. Microbiol. 2, 16251 (2017).

17. Jimmy, S. et al. A widespread toxin-antitoxin system exploiting growth control via alarmone signaling. Proc. Natl. Acad. Sci. 117, 10500-10510 (2020).

18. Vasu, K. \& Nagaraja, V. Diverse Functions of Restriction-Modification Systems in Addition to Cellular Defense. Microbiol. Mol. Biol. Rev. 77, 53-72 (2013).

19. Deveau, H., Garneau, J. E. \& Moineau, S. CRISPR/Cas System and Its Role in Phage-Bacteria Interactions. Annu. Rev. Microbiol. 64, 475-493 (2010).

20. Chopin, M. C., Chopin, A. \& Bidnenko, E. Phage abortive infection in lactococci: Variations on a theme. Curr. Opin. Microbiol. 8, 473-479 (2005).

21. Otsuka, Y., Koga, M., Iwamoto, A. \& Yonesaki, T. A role of RnIA in the RNase LS activity from 
bioRxiv preprint doi: https://doi.org/10.1101/2020.06.22.160242; this version posted June 22, 2020. The copyright holder for this preprint (which was not certified by peer review) is the author/funder, who has granted bioRxiv a license to display the preprint in perpetuity. It is made available under aCC-BY 4.0 International license.

Escherichia coli. Genes Genet. Syst. 82, 291-299 (2007).

22. Otsuka, Y. \& Yonesaki, T. Dmd of bacteriophage T4 functions as an antitoxin against Escherichia coli LsoA and RnIA toxins. Mol. Microbiol. 83, 669-681 (2012).

23. Elfenbein, J. R. et al. Multicopy Single-Stranded DNA Directs Intestinal Colonization of Enteric Pathogens. PLoS Genet. 11, 1-24 (2015).

24. Bobonis, J. et al. Bacterial retrons encode tripartite toxin/antitoxin systems. Preprint at bioRxiv (2020).

25. Saka, K. et al. A complete set of Escherichia coli open reading frames in mobile plasmids facilitating genetic studies. DNA Res. 12, 63-68 (2005).

26. Otsuka, Y. et al. GenoBase: Comprehensive resource database of Escherichia coli K-12. Nucleic Acids Res. 43, D606-D617 (2015).

27. Simon, A. J., Ellington, A. D. \& Finkelstein, I. J. Retrons and their applications in genome engineering. Nucleic Acids Res. 47, 11007-11019 (2019).

28. Hattman, S. et al. Common evolutionary origin of the phage T4 dam and host Escherichia coli dam DNAadenine methyltransferase genes. J. Bacteriol. 164, 932-937 (1985).

29. Marinus, M. G. \& Morris, N. R. Isolation of deoxyribonucleic acid methylase mutants of Escherichia coli K12. J. Bacteriol. 114, 1143-1150 (1973).

30. Marinus, M. G. \& Løbner-Olesen, A. DNA Methylation. EcoSal Plus 6, 1-62 (2014).

31. Torreblanca, J. \& Casadesús, J. DNA adenine methylase mutants of Salmonella typhimurium and a novel dam- regulated locus. Genetics 144, 15-26 (1996).

32. Sternberg, N. \& Coulby, J. Cleavage of the bacteriophage P1 packaging site (pac) is regulated by adenine methylation. Proc. Natl. Acad. Sci. U. S. A. 87, 8070-8074 (1990).

33. Lampson, B. C., Inouye, M. \& Inouye, S. Retrons, msDNA, and the bacterial genome. Cytogenet. Genome Res. 110, 491-499 (2005).

34. Jung, H., Liang, J., Jung, Y. \& Lim, D. Characterization of cell death in Escherichia coli mediated by XseA, a large subunit of exonuclease VII. J. Microbiol. 53, 820-828 (2015).

35. Geier, G. E. \& Modrich, P. Recognition sequence of the dam methylase of Escherichia coli K12 and mode of cleavage of Dpn I endonuclease. J. Biol. Chem. 254, 1408-1413 (1979).

36. Szyf, M. et al. DNA methylation pattern is determined by the intracellular level of the methylase. Proc. Natl. Acad. Sci. 81, 3278-3282 (1984).

37. Joseph, J. W. \& Kolodner, R. Exonuclease VIII of Escherichia coli. II. Mechanism of action. J. Biol. Chem. 258, 10418-10424 (1983).

38. Kirchner, J., Lim, D., Witkin, E. M., Garvey, N. \& Roegner-Maniscalco, V. An SOS-inducible defective retronphage ( $\varphi$ R86) in Escherichia coli strain B. Mol. Microbiol. 6, 2815-2824 (1992).

39. Hsu, M. Y., Inouye, S. \& Inouye, M. Structural requirements of the RNA precursor for the biosynthesis of the branched RNA-linked multicopy single-stranded DNA of Myxococcus xanthus. J. Biol. Chem. 264, 6214-6219 (1989).

40. Kato, F., Yoshizumi, S., Yamaguchi, Y. \& Inouye, M. Genome-wide screening for identification of novel toxin-antitoxin systems in Staphylococcus aureus. Appl. Environ. Microbiol. 85, (2019).

41. Brochado, A. R. \& Typas, A. High-throughput approaches to understanding gene function and mapping network architecture in bacteria. Curr. Opin. Microbiol. 16, 199-206 (2013).

42. Koo, B.-M. et al. Construction and Analysis of Two Genome-Scale Deletion Libraries for Bacillus subtilis. Cell Syst. 4, 291-305.e7 (2017).

43. Cui, L. et al. A CRISPRi screen in E. coli reveals sequence-specific toxicity of dCas9. Nat. Commun. 9, 1912 (2018).

44. Liu, X. et al. High-throughput CRISPRi phenotyping identifies new essential genes in Streptococcus 
bioRxiv preprint doi: https://doi.org/10.1101/2020.06.22.160242; this version posted June 22, 2020. The copyright holder for this preprint (which was not certified by peer review) is the author/funder, who has granted bioRxiv a license to display the preprint in perpetuity. It is made available under aCC-BY 4.0 International license.

pneumoniae . Mol. Syst. Biol. 13, 931 (2017).

45. Keseler, I. M. et al. The EcoCyc database: Reflecting new knowledge about Escherichia coli K-12. Nucleic Acids Res. 45, D543-D550 (2017).

46. Millman, A. et al. Bacterial retrons function in anti-phage defense. Preprint at bioRxiv (2020).

47. Hattman, S. DNA methylation of T-even bacteriophages and of their nonglucosylated mutants: Its role in P1-directed restriction. Virology 42, 359-367 (1970).

48. Handa, N. \& Kobayashi, I. Type III restriction is alleviated by bacteriophage (RecE) homologous recombination function but enhanced by bacterial (RecBCD) function. J. Bacteriol. 187, 7362-7373 (2005).

49. Makarova, K. S., Anantharaman, V., Aravind, L. \& Koonin, E. V. Live virus-free or die: coupling of antivirus immunity and programmed suicide or dormancy in prokaryotes. Biol. Direct 7, 40 (2012).

50. Christensen, S. K., Mikkelsen, M., Pedersen, K. \& Gerdes, K. RelE, a global inhibitor of translation, is activated during nutritional stress. Proc. Natl. Acad. Sci. 98, 14328-14333 (2001).

51. Christensen, S. K. et al. Overproduction of the Lon protease triggers inhibition of translation in Escherichia coli: involvement of the yefM-yoeB toxin-antitoxin system. Mol. Microbiol. 51, 1705-1717 (2004).

52. Christensen-Dalsgaard, M., Jørgensen, M. G. \& Gerdes, K. Three new RelE-homologous mRNA interferases of Escherichia coli differentially induced by environmental stresses. Mol. Microbiol. 75, 333348 (2010).

53. Page, R. \& Peti, W. Toxin-antitoxin systems in bacterial growth arrest and persistence. Nat. Chem. Biol. 12, 208-214 (2016).

54. Maisonneuve, E., Castro-Camargo, M. \& Gerdes, K. RETRACTED: (p)ppGpp Controls Bacterial Persistence by Stochastic Induction of Toxin-Antitoxin Activity. Cell 154, 1140-1150 (2013).

55. Germain, E., Roghanian, M., Gerdes, K. \& Maisonneuve, E. RETRACTED: Stochastic induction of persister cells by HipA through (p)ppGpp-mediated activation of mRNA endonucleases. Proc. Natl. Acad. Sci. 112, 5171-5176 (2015).

56. Pedersen, K. \& Gerdes, K. Multiple hok genes on the chromosome of Escherichia coli. Mol. Microbiol. 32 , 1090-1102 (1999).

57. Wilbaux, M., Mine, N., Guérout, A.-M., Mazel, D. \& Van Melderen, L. Functional Interactions between Coexisting Toxin-Antitoxin Systems of the ccd Family in Escherichia coli O157:H7. J. Bacteriol. 189, 2712-2719 (2007).

58. LeRoux, M., Culviner, P. H., Liu, Y. J., Littlehale, M. L. \& Laub, M. T. Stress Can Induce Transcription of Toxin-Antitoxin Systems without Activating Toxin. Mol. Cell 1-13 (2020).

59. Song, S. \& Wood, T. K. Toxin/Antitoxin System Paradigms: Toxins Bound to Antitoxins Are Not Likely Activated by Preferential Antitoxin Degradation. Advanced Biosystems 4, 1900290 (2020).

60. Goormaghtigh, F. et al. Reassessing the Role of Type II Toxin-Antitoxin Systems in Formation of Escherichia coli Type II Persister Cells. MBio 9, 1-14 (2018).

61. Harms, A., Fino, C., Sørensen, M. A., Semsey, S. \& Gerdes, K. Prophages and Growth Dynamics Confound Experimental Results with Antibiotic-Tolerant Persister Cells. MBio 8, 1-18 (2017).

62. Fineran, P. C. et al. The phage abortive infection system, ToxIN, functions as a protein-RNA toxinantitoxin pair. Proc. Natl. Acad. Sci. U. S. A. 106, 894-899 (2009).

63. Van Melderen, L. Toxin-antitoxin systems: Why so many, what for? Curr. Opin. Microbiol. 13, 781-785 (2010).

64. Yee, T., Furuichi, T., Inouye, S. \& Inouye, M. Multicopy single-stranded DNA isolated from a gramnegative bacterium, Myxococcus xanthus. Cell 38, 203-209 (1984). 
612

613

614

615

616

617

618

619

620

621

622

623

624

625

626

627

628

629

630

631

632

633

634

635

636

637

638

639

640

641

642

643

644

645

646

647

648

METHODS

\section{Bacterial strains, plasmids, primers, and growth conditions}

All bacterial strain genotypes, plasmids, plasmid construction, and primers used in this study are described in Tables S3-S6, respectively. Bacteria were grown in Lysogeny Broth Lennox (LB-Lennox; Tryptone $10 \mathrm{~g} / \mathrm{L}$, Yeast Extract $5 \mathrm{~g} / \mathrm{L}$, Sodium Chloride $5 \mathrm{~g} / \mathrm{L}$ ). LB-Agar plates (LB plates) were prepared by adding separately-autoclaved $2 \%$ molten-Agar in liquid LB. All plasmid-carrying bacterial strains were streaked-out, grown, and assayed with appropriate antibiotics to maintain the plasmids. Plasmids carrying PBAD-promoters were induced with $0.2 \% \mathrm{D}$-arabinose, while plasmids carrying Ptac promoters were induced with low $(0.1 \mathrm{mM})$ or high (1 mM) concentrations of isopropyl $\beta$-D-1-thiogalactopyranoside (IPTG). Bacterial strains with chromosomally-inserted antibiotic-resistance cassettes were streaked-out from stocks on antibiotic-LB plates, but grown/assayed thereafter without antibiotics. Antibiotics used were Spectinomycin $(100 \mu \mathrm{g} / \mathrm{mL})$, Ampicillin $(50 \mu \mathrm{g} / \mathrm{mL})$, Tetracycline $(10 \mu \mathrm{g} / \mathrm{mL})$, and Kanamycin (30 $\mathrm{\mu g} / \mathrm{mL})$. For diaminopimelic acid (DAP) auxotroph-strains, DAP was provided at a final concentration of $0.3 \mathrm{mM}$. Cold-sensitive strains (STm retron-Sen2 mutants) were freshly streaked-out from glycerol stocks and kept only at $37^{\circ} \mathrm{C}$ before every experiment, in order to avoid suppressor mutations. For cold-sensitivity growth tests, strains were incubated at $20^{\circ} \mathrm{C}$, or at $15^{\circ} \mathrm{C}$, for 48 hours, or 72 hours, respectively.

\section{Toxin Inhibition/Activation Conjugation (TIC/TAC) procedure}

384-colony-arrays of the MOB plasmid library (carried within an E. coli F+ strain; JA200 ${ }^{25}$ ) and of the TransBac plasmid library (carried within an E. coli $\mathrm{F}^{+}$dapA strain; BW38029 ${ }^{26}$ ) were pinned from liquid glycerol-stocks to ampicillin-LB and tetracycline-DAP-LB plates, respectively, using a Singer ROTOR and 384-density long-pin Singer RePads, and were grown overnight. Conjugation recipient strains (E. coli BW25113 ${ }^{66}$ ), carried either a p-rcaT plasmid (for TIC), or a p-retron plasmid (for TAC), which both contain a spectinomycin resistance cassette (plasmids detailed in Tables S4-S5). Recipient strains were grown overnight in spectinomycin-LB, and $200 \mu \mathrm{L}$ of cultures (diluted to $\mathrm{OD}_{595}=0.5$ ) were spread using glass beads on LB plates (for MOB), or on LB-DAP plates (for TransBac). Plates with recipient-lawns were incubated in a non-humid incubator at $37^{\circ} \mathrm{C}$ for 1 hour. Next, 384-colonyarrays of the donor-libraries were pinned on top of the recipient-lawns, using 384 short-pin Singer RePads. Donor and recipients were allowed to conjugate for 8 hours in a humid incubator at $37^{\circ} \mathrm{C}$. Subsequently, cells from the conjugation plates were pinned onto doubleantibiotic-selection plates, using 384 short-pin Singer RePads, in order to select for BW25113 transconjugants carrying both plasmids ( $\mathrm{p}$-rcaT/p-retron + library-plasmids). Double-selection plates contained either ampicillin-spectinomycin, or tetracycline-spectinomycin, for MOB or TransBac libraries, respectively, and transconjugants were grown for 24 hours at $37^{\circ} \mathrm{C}$. 
Transconjugants were subjected to a second round of selection on double-antibiotic plates, and were also re-arrayed in a 1536-colony format. 1536-colony transconjugant plates were incubated for 10 hours at $37^{\circ} \mathrm{C}$, and then each plate was pinned (using 1536-density short-pin Singer RePads) on two replicates of double-antibiotic selection plates (third-round of selection; "source-plates"). Source plates were incubated for 5 hours at $37^{\circ} \mathrm{C}$ and were used to pin onto double-selection LB-plates ("test-plates"), using 1536-density short-pin RePads. Test-plates contained either no inducer, only arabinose, only IPTG (low or high), or combinations of both (TIC TransBac screen was performed only with low IPTG concentrations). Test-plates were incubated for 13 hours at $37^{\circ} \mathrm{C}$, and afterwards imaged using a Canon EOS Rebel T3i camera under controlled light settings (S\&P robotics).

\section{TIC/TAC data analysis}

Bacterial colony morphological features for each strain were quantified by using the Iris imageanalysis platform ${ }^{65}$, and colony integral opacity values were used as a fitness proxy. To account the effects of plasmid induction on fitness, we used plates containing only low or high concentrations of IPTG as controls (control plates). These were compared to plates in which the library-plasmids and the p-rcaT/p-retron were co-induced with IPTG and arabinose (experiment plates). For quality control, we empirically derived cut-offs for strains that were a) growth-inhibited in the control plates (opacity values $<50,000$ ), b) mucoid in the control plates (colony densities of both replicates $>51^{65}$ ), and c) noisy strains in control and/or experiment plates (standard deviation for opacity values $>23,000$ - median opacities were: TAC control 103,820, TAC experiment - 71,680, TIC control - 106,941, TIC experiment - 24,357). Strains exceeding any of the three cut-offs were flagged and removed from the final reported dataset, but visible on Table S2. Plate exterior opacity values (four outermost rows and columns) were each multiplicatively corrected to match the mean growth of the interior of the plate. Plate-toplate biases were also multiplicatively corrected to the same mean. Subsequently, z-scores of those corrected opacity values were calculated per condition, and mean z-scores were calculated per mutant across technical replicates. The final reported score is calculated as the difference between the mean z-scores of each mutant in the experiment and the control plates. All raw and processed data from the TIC/TAC analysis can be found in Table S2.

\section{TIC/TAC validation procedure}

To test candidate genes for blocker/trigger activity, individual conjugation donor strains were single-colony purified from the MOB ${ }^{25}$ and TransBac ${ }^{26}$ libraries, and used to construct new transconjugants that were assayed through colony-array and spot growth-tests. To verify that the plasmids contained the appropriate open reading frames, the plasmids were isolated and sequenced. 
686 For colony-array growth tests, MOB donor strains (JA200 ${ }^{25}$ ) or TransBac donor strains 687 (BW38029 ${ }^{26}$ ) were grown overnight in $600 \mu \mathrm{L}$ of $\mathrm{LB}$ at $37^{\circ} \mathrm{C}$ in a $1 \mathrm{~mL}$-volume 96 -deep-well 688 plate (Thermo Scientific; catalogue number 260251), aliquoted in 96-well plates with 15\% 689 glycerol, and kept at $-80^{\circ} \mathrm{C}$ until further use. The 96-colony-array donor strains were 690 conjugated with E. coli BW25113 recipient-strains carrying plasmids p-empty, p-retron, p691 retron- $\Delta r c a T$, and $p-r c a T$ in four separate LB agar plates (conjugation as described in the 692 TIC/TAC procedure section). Subsequently, the transconjugant strains were combined in a 693 single 384-array, and pinned on plates containing either no inducer, only arabinose, only IPTG 694 (low or high), or combinations of both. Plates were incubated for 15 hours in a humid-incubator 695 at $37^{\circ} \mathrm{C}$, and the 384 -density arrayed plates were imaged as described before. Pictures were 696 analysed using Iris ${ }^{65}$, and integral opacity values were used to calculate fitness scores. Fitness 697 scores for TAC were calculated per condition (per plate) as the opacity ratio between E. coli 698 strains carrying $\mathrm{p}$-retron- $\Delta r c a T$ and $\mathrm{p}$-retron (and trigger-plasmid-X). Fitness scores for TIC 699 were calculated per-IPTG-condition as the opacity ratio between an $E$. coli strain carrying $p$ 700 rcaT (and blocker-plasmid-X) and the average opacity value of strains carrying $\mathrm{p}$-rcaT (and 701 trigger-plasmid- $X$, as negative control).

702

For spot growth-tests, purified MOB and TransBac library-plasmids were first transformed in MFDpir 67 and BW38029 26 conjugation donor-strains, respectively. Next, donors were conjugated with either $E$. coli BW25113 carrying plasmids p-retron- $\Delta$ rcaT and p-retron (for TAC), or E. coli BW25113 carrying plasmid p-rcaT (for TIC). Conjugation was carried out as described in the plasmid conjugation section. Strains were spotted on plates containing either no inducer, only arabinose, only IPTG (low or high), or combinations of both, and spot growth tests were carried out as described below.

\section{Spot growth tests}

712 Single bacterial colonies were inoculated in $2 \mathrm{~mL}$ of $\mathrm{LB}$, and incubated at $37^{\circ} \mathrm{C}$ for $5-6$ hours 713 in a roller drum (until an OD595 of 5-6). Cultures were then stepwise serially-diluted (ten-fold) 714 eight times in LB $(100 \mu \mathrm{L}$ culture $+900 \mu \mathrm{L}$ LB). Subsequently, using a 96-pinner (V\&P Scientific, 715 catalogue number: VP 404), $\sim 10 \mu \mathrm{L}$ of culture dilutions were spotted on LB plates containing 716 appropriate antibiotics, arabinose, and/or low/high concentrations of IPTG, when applicable. LB plates were grown at appropriate temperatures as described above.

\section{Genetic techniques}

Non-mobilizable plasmids were introduced in E. coliBW25113 ${ }^{66}$ and MFDpir ${ }^{67}$ strains by TSS

721 transformation ${ }^{68}$, whereas STm strains were transformed by electroporation ${ }^{69}$. Mobilizable 722 plasmids were introduced in bacterial strains by conjugation (described in the Plasmid 
conjugation section below). To construct the p-retron ${ }^{\text {mut }}$ plasmid (msd: GATC $\rightarrow$ GTTC), the source plasmid ( $p$-retron ${ }^{\mathrm{WT}}$ ) was mutagenized through PCR using a kit (NEB; Q5-Site-Directed Mutagenesis Kit, catalogue number E0554S), by following the instructions of the manufacturer. Mutagenic primers used were JB433 and JB434 (primers detailed in Table S6).

\section{Plasmid conjugation}

Mobilizable plasmids were introduced to $E$. coli or STm strains through conjugation (donors were either E. coli JA200 ${ }^{25}$, E. coli BW28029 ${ }^{26}$ or E. coli MFDpir ${ }^{67}$ strains). Conjugation was carried out by growing single colonies of both recipient and donor strains in LB overnight at $37^{\circ} \mathrm{C}$ in a drum-roller (LB supplemented with DAP or appropriate antibiotics where applicable). Subsequently, $200 \mu \mathrm{L}$ of diluted overnight cultures $\left(\mathrm{OD}_{595}=0.5\right)$ of the donor strains were spread on LB-plates (supplemented with DAP if applicable), and plates were incubated at $37^{\circ} \mathrm{C}$ in a dry incubator for 1 hour. Next, $10 \mu \mathrm{L}$ of diluted overnight cultures (OD595=0.5) of the recipient strains were spotted on top of the lawn of the donor strain, and the conjugation plates were incubated for 6 hours at $37^{\circ} \mathrm{C}$. Finally, transconjugant strains were selected by streaking them out from the area where the recipient strains were spotted, in either double-antibiotic selection plates, or single-antibiotic plates but without supplementing them with DAP (if applicable). Selection plates were grown overnight at $37^{\circ} \mathrm{C}$, and transconjugants were singlecolony purified for further use. The high-throughput conjugation protocol is described in the TIC/TAC procedure section.

\section{msDNA purification, Dpnl/RecE digestion, denaturing electrophoresis, and silver-stain} msDNA was extracted by the alkaline lysis method, as described in ${ }^{24}$. To assess the methylation status of msDNA, the extracted msDNA was further purified from plasmid DNA contaminants by the crush and soak method ${ }^{70}$. Briefly, msDNA extracts (from $200 \mathrm{~mL}$ of bacterial cultures) were electrophoresed in 12\% TBE-polyacrylamide gels ( $70 \mathrm{~V}$ for 4.5 hours). The equivalent of extracted msDNA from $60 \mathrm{~mL}$ of culture were loaded per well, in order to increase the efficiency of subsequent elution from the acrylamide gel. Gels were stained with ethidium bromide, and the msDNA-containing gel slices were transferred to Eppendorf tubes. Next, the gel-slices were crushed against the walls of the tubes with a tip, suspended in two gel-slice volumes of acrylamide elution buffer ${ }^{70}$, vortexed, and incubated at $37^{\circ} \mathrm{C}$ in a tabletop roller for 16 hours. Samples were centrifuged at $14,000 \mathrm{rpm} / 5 \mathrm{~min} / \mathrm{RT}$, and the supernatants were transferred to fresh tubes. An equal volume of isopropanol was added to the supernatants, samples were vortexed, and msDNA was precipitated overnight at $4^{\circ} \mathrm{C}$. Next, samples were centrifuged at $14,000 \mathrm{rpm} / 60 \mathrm{~min} / 4^{\circ} \mathrm{C}$, pellets were washed once with 1 $\mathrm{mL}$ of $70 \%$ ethanol, and washed pellets were centrifuged again at $14,000 \mathrm{rpm} / 60 \mathrm{~min} / 4^{\circ} \mathrm{C}$. Pellets (purified msDNA) were air-dried for 15 minutes, and resuspended in $12 \mu \mathrm{L}$ of distilled 
water. Subsequently, 1 unit/ $\mu \mathrm{L}$ of Dpnl (NEB; catalogue number R0176S) was added in $5 \mu \mathrm{L}$ of purified msDNA and digested overnight at $37^{\circ} \mathrm{C}$. In parallel, $5 \mu \mathrm{L}$ of purified msDNA were incubated in the same buffer (without Dpnl), and msDNA-digests were electrophoresed in a denaturing $20 \%$ urea-TBE-polyacrylamide gel, as described in ${ }^{71}$. Briefly, $2 x$ formamide loading buffer ( $90 \%$ formamide, $0.5 \%$ EDTA, $0.1 \%$ xylene cyanol, $0.1 \%$ bromophenol blue) was added to the msDNA-digests, and samples were heated to $95^{\circ} \mathrm{C}$ for $10 \mathrm{~min}$, and were quickly transferred in ice. The denaturing gel was pre-ran for 1 hour at $55^{\circ} \mathrm{C}$, and subsequently msDNA-digests were electrophoresed for 3.5 hours, with constant voltage (60 V). Finally, DNA were stained with silver by using a silver-stain kit (Roth; article number L533.1), following the procedure as described in ${ }^{72}$.

For digesting msDNA with exodeoxyribonuclease VIII (RecE), msDNA were extracted as described in ${ }^{24}$, and msDNA extracts were incubated overnight at $37^{\circ} \mathrm{C}$ with 0.5 units $/ \mu \mathrm{L}$ of truncated RecE (NEB; catalogue number M0545S). Subsequently, msDNA-digests were electrophoresed in $12 \%$ TBE-Polyacrylamide gels, and stained with ethidium bromide for visualization.

\section{Statistical analyses}

For the analysis of TIC/TAC data, z-score difference means per overexpression strain were calculated as the average z-score difference across clones and replicates, separately for each overexpression library. One-tailed p-values (p.value; Table S2) were calculated for each overexpression strain per induction condition, by using the probability distribution function for the normal distribution. Parameters used were the means and standard deviations of the score distribution of each condition. One-tailed FDR-corrected p-values (q.value; Table S2) were subsequently calculated using the Benjamini-Hochberg method ${ }^{73}$. For the aggregated prophage genes enrichment analysis (Table S1), hit genes were pooled across the two libraries, and $p$-values for prophage-gene enrichment were calculated using the Fisher's exact test. Since both MOB ${ }^{25}$ and TransBac ${ }^{26}$ libraries are derived from E. coli, only unique genes from the two libraries were accounted for in the background frequency, for the aggregated test. A similar Fisher's test was also conducted per induction condition, separately for the two libraries (Table S1). Flagged strains were not included in the prophage gene enrichment analyses. E. coli BW25113 and E. coli BL21 (DE3) genes were annotated as prophage genes by using the PHASTER database ${ }^{74}$. 


\section{EXTENDED DATA FIGURE LEGENDS}

795 Extended Data Figure 1. Low-IPTG induction, reproducibility, and hits heatmap for TAC.

796 (A-B) TAC results from low-IPTG induction. Experiments as described in Fig. 1B-C, but with low-IPTG plasmid-library induction for MOB (A; p1) ${ }^{25}$ and TransBac (B; p2) ${ }^{26}$.

(C) TAC reproducibility in control and experiment plates. Unprocessed opacity values of strains against each other. The overexpression library and IPTG concentration contained in each plate-pair are denoted above the plots. Coefficients of determination $\left(R^{2}\right)$ are shown for each reproducibility plot.

803 (D) Non-parametric comparison of identified triggers across overexpression libraries. Trigger804 genes identified from the MOB ${ }^{25}(p 1)$ and the $\operatorname{TransBac}^{26}(\mathrm{p} 2)$ overexpression libraries were rank-ordered in percentiles based on their mean z-score difference value, as calculated from TAC screens conducted in low, or high-IPTG induction. Not available (NA) denotes genes for which measurements were flagged as problematic (see Methods) in the respective library/IPTG-concentration. Absent denotes genes which are absent from one library. Phagerelated trigger-genes are in bold.

Extended Data Figure 2. Validation of trigger genes from TAC screens. Triggers specifically inhibit growth by activating RcaT, with triggering degree depending on libraryplasmid induction levels.

(A) Plasmids (p1/p2-trigger gene in green) were conjugated into E. coli BW25113 carrying plasmids p-empty, p-retron, p-retron- $\Delta$ rcaT, or p-rcaT. 384-colony-arrays of the transconjugants were pinned on LB plates containing appropriate antibiotics, arabinose, and IPTG concentrations (no IPTG, low, high). The y-axis represents the triggering-degree of each plasmid, measured as the colony opacity ratio of strains (p-retron- $\Delta r c a T+p 1 / p 2$-trigger) divided by the ( $p$-retron $+\mathrm{p} 1 / \mathrm{p} 2$-trigger). $\mathrm{p} 1$-control values were derived by measuring the same colony opacity ratio, for $\mathrm{p} 1$-blocker genes ( $n=72 ; 36$ biological $\mathrm{X} 2$ technical replicates), as a negative control. Ratios were calculated from $n=10-5$ biological $X 2$ technical replicates for $\mathrm{p} 1$-trigger genes (except for $\mathrm{p} 1-\mathrm{yfb} O ; n=8-4$ biological $\mathrm{X} 2$ technical replicates), and from $n=24-12$ biological $X 2$ technical replicates for p2-trigger genes. Representative colonies of strains carrying p1/p2-trigger plasmids are shown below the graphs. Horizontal bars denote the average fitness ratio, and error bars denote the standard deviation. Grey horizontal bars denote the p1-control fitness ratio. p1-dam was plotted separately, to avoid compressing the scores of the rest of the trigger-genes.

(B) Plasmids (p1/p2-trigger gene in green, and p1-empty/p2-empty) from conjugation donor strains were conjugated with $E$. coli BW25113 carrying plasmids p-retron- $\Delta r c a T$ and p-retron. 
831

832

833

834

835

836

837

838

839

840

841

842

843

844

845

846

847

848

849

850

851

852

853

854

855

856

857

858

859

860

861

862

863

864

865

866

spotted on LB plates containing antibiotics, arabinose, and IPTG (no IPTG, low, or high), and incubated at $37^{\circ} \mathrm{C}$.

Extended Data Figure 3. High-IPTG induction, reproducibility, and hits heatmap for TIC. (A) TIC results from high-IPTG induction. Experiments as described in Fig. 1D, but with lowIPTG plasmid-library induction for $\operatorname{MOB}(A ; p 1)^{25}$. Gene yfbO was selected as a blocker, despite not passing the significance cut-off, due to genetic linkage with $y f b N$.

(B) TIC reproducibility in control and experiment plates - as in ED Fig. $1 C$.

(C) Non-parametric comparison of identified blockers across overexpression libraries - as in ED Fig. 1D. Grey-colored genes are presumably affecting induction and/or conjugation levels. Phage-related trigger-genes are in bold.

\section{Extended Data Figure 4. Validation of blocker genes from TIC screens.}

Blockers specifically alleviate RcaT-mediated toxicity with blocking degree depending on library-plasmid induction levels.

(A) Plasmids (p1-blocker gene in blue and p1-empty in black) were conjugated into $E$. coli BW25113 carrying plasmids p-empty, p-retron, p-retron- $\Delta$ rcaT, or p-rcaT. 384-colony-arrays of the transconjugants were pinned on LB plates containing appropriate antibiotics, arabinose, and IPTG concentrations (no IPTG, low, or high). The y-axis represents the blocking degree of each plasmid, measured as the colony opacity ratio of strains carrying the different blockers $(p-r c a T+p 1$-blocker) divided by the average of a strain carrying trigger plasmids $(p-r c a T+p 1-$ trigger), as a negative control. Fitness ratios calculated from $n=8-4$ biological $X 2$ technical replicates. The mean colony opacity for $\mathrm{p} 1$-trigger was derived from $n=110-55$ biological $X 2$ technical replicates. Representative colonies of strains carrying p1-blocker plasmids shown below the graphs. Horizontal bars denote the average fitness ratio, and error bars denote the standard deviation. Grey horizontal bar denotes the expected fitness ratio in the absence of blocking effects.

(B) Plasmids (p1/p2-blocker gene in blue, and p1-empty/p2-empty) from conjugation donor strains were conjugated with E. coli BW25113 carrying plasmid p-rcaT or a p1-empty plasmid. Transconjugants were grown as in ED Fig. 2B.

\section{Extended Data Figure 5. Dam-mediated retron-TA triggering.}

(A) Bacterial/phage dam homologues trigger the retron-TA. E. coli BW25113 carrying combinations of plasmids p-retron- $\Delta r c a T$, p-retron, p1-dam ${ }^{\mathrm{Ec}}, \mathrm{p} 1-$ dam $^{\mathrm{STm}}, \mathrm{p} 1-\mathrm{dam}^{\mathrm{P} 1}$, and p1empty, were grown for 5 hours in LB with appropriate antibiotics, serially diluted, spotted on LB plates with antibiotics, with/without arabinose, and IPTG (low), and incubated at $37^{\circ} \mathrm{C}$. 
867

868

869

870

871

872

873

874

875

876

877

878

879

880

881

882

883

884

885

886

887

888

889

890

891

892

893

894

895

896

897

898

899

(B) $r c a T$ in p-retron ${ }^{\text {mut }}$ is functional and expressed. E. coli BW25113 $\Delta x \operatorname{se} A$ strains carrying combinations of plasmids $\mathrm{p}$-retron ${ }^{\mathrm{WT}}, \mathrm{p}$-retron ${ }^{\mathrm{mut}}, \mathrm{p} 1$-dam, and $\mathrm{p} 1$-empty, were grown and spotted as described in panel A. Representative data shown from two independent experiments.

(C) p-retron ${ }^{\text {mut }}$ produces msDNA at equivalent levels as wildtype retron. msDNA was extracted from STm strains carrying $p$-retron ${ }^{W T}$, or p-retron ${ }^{m u t}$. Plasmids were induced with arabinose. Extracted msDNA was electrophoresed in a TBE-Polyacrylamide gel.

Extended Data Figure 6. racC-recE constitute a blocker-trigger gene-pair for retronEco9.

(A) RecE triggers the retron-Eco9. E. coli BW25113 carrying combinations of plasmids pretron-Eco9, p-retron- $\Delta r c a T$-Eco9, p1-recE, and p1-empty, were grown for 5 hours in LB with appropriate antibiotics, serially diluted, spotted on LB plates with antibiotics, arabinose, and IPTG (low), and incubated at $37^{\circ} \mathrm{C}$. Representative data shown from three independent experiments.

(B) RecE degrades mature and immature msDNA in vitro. msDNA was extracted from STm strains (WT, or $\Delta x s e A$ ) carrying plasmid p-retron- $\Delta r c a T$. msDNA extracts were incubated with recombinant RecE (see Methods), and digests were electrophoresed on TBE-Polyacrylamide gels.

(C) RacC also blocks RcaT-Eco9. E. coli BW25113 carrying combinations of plasmids p-rcaTEco9, p1-racC, and p1-empty, were grown and spotted as in panel A with different IPTG concentrations.

\section{Extended Data Figure 7. RT-Eco1 partially triggers endogenous STm retron-TA.}

STm strains (WT, $\Delta$ retron, $\Delta r r t T, \Delta m s r m s d$ ) carrying plasmid p2-RT-Eco1 were arrayed on LB plates containing tetracycline and IPTG (high), and plates were incubated either at $15^{\circ} \mathrm{C}, 20^{\circ} \mathrm{C}$, or $37^{\circ} \mathrm{C}$. The $y$-axis represents the triggering degree of RT-Eco1, measured as the colony size ratio of strain ( $\Delta$ retron + p2-RT-Eco1) divided by the $(\mathrm{WT}+\mathrm{p} 2-\mathrm{RT}-\mathrm{Ec01})$. Fitness ratios calculated from $n=24-12$ biological X 2 technical replicates. Representative STm colonies carrying p2-RT-Eco1 are shown below the graph. Horizontal bars denote the average fitness ratio, and error bars denote standard deviation. Grey bar denotes the expected fitness ratio in the absence of triggering effects. 
900

901

902

903

904

905

906

907

908

909

910

911

912

913

914

915

916

917

918

919

920

921

922

923

924

925

926

927

928

929

930

931

\section{SUPPLEMENTARY INFORMATION}

Supplementary Table 1. Prophage genes enrichment in TIC/TAC hit genes.

Supplementary Table 2. Raw and processed TIC/TAC fitness data.

Supplementary Table 3. Genotypes of bacterial strains used in this study.

Supplementary Table 4. Description of plasmids used in this study.

Supplementary Table 5. Description of construction of plasmids used in this study.

Supplementary Table 6. List of primers used in this study.

\section{REFERENCES METHODS AND EXTENDED FIGURE LEGENDS}

65. Kritikos, G. et al. A tool named Iris for versatile high-throughput phenotyping in microorganisms. Nat.

66. Baba, T. et al. Construction of Escherichia coli K-12 in-frame, single-gene knockout mutants: The Keio Microbiol. 2, 17014 (2017).

collection. Mol. Syst. Biol. 2, 2006.0008 (2006).

67. Ferrières, L. et al. Silent mischief: Bacteriophage Mu insertions contaminate products of Escherichia coli random mutagenesis performed using suicidal transposon delivery plasmids mobilized by broad-hostrange RP4 conjugative machinery. J. Bacteriol. 192, 6418-6427 (2010).

68. Chung, C. T., Niemela, S. L. \& Miller, R. H. One-step preparation of competent Escherichia coli: transformation and storage of bacterial cells in the same solution. Proc. Natl. Acad. Sci. 86, 2172-2175 (1989).

69. Chassy, B. Transformation of bacteria by electroporation. Trends Biotechnol. 6, 303-309 (1988).

70. Green, M. R. \& Sambrook, J. Isolation of DNA Fragments from Polyacrylamide Gels by the Crush and Soak Method. Cold Spring Harb. Protoc. 2019, pdb.prot100479 (2019).

71. Summer, H., Grämer, R. \& Dröge, P. Denaturing Urea Polyacrylamide Gel Electrophoresis (Urea PAGE). J. Vis. Exp. 3-5 (2009).

72. Bassam, B. J. \& Gresshoff, P. M. Silver staining DNA in polyacrylamide gels. Nat. Protoc. 2, 2649-2654 (2007).

73. Benjamini, Y. \& Hochberg, Y. Controlling the False Discovery Rate: A Practical and Powerful Approach to Multiple Testing. J. R. Stat. Soc. Ser. B 57, 289-300 (1995).

74. Arndt, D. et al. PHASTER: a better, faster version of the PHAST phage search tool. Nucleic Acids Res. 44, W16-W21 (2016).

75. Uehara, T., Parzych, K. R., Dinh, T. \& Bernhardt, T. G. Daughter cell separation is controlled by cytokinetic ring-activated cell wall hydrolysis. EMBO J. 29, 1412-1422 (2010). 
bioRxiv preprint doi: https://doi.org/10.1101/2020.06.22.160242.this version posted June 22, 2020. The copyright holder for this preprint (which was not certified by peer review) is the author/funder, w made available under aCES 4.0 International license.

A

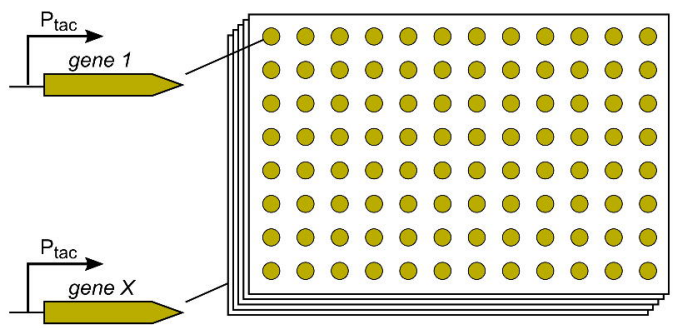

$+$
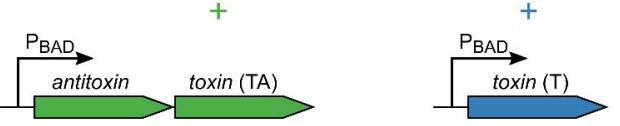

Toxin Activation Conjugation

(TAC)

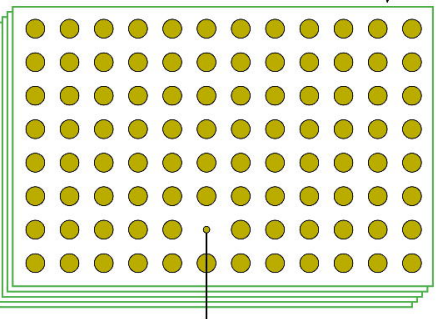

TA trigger

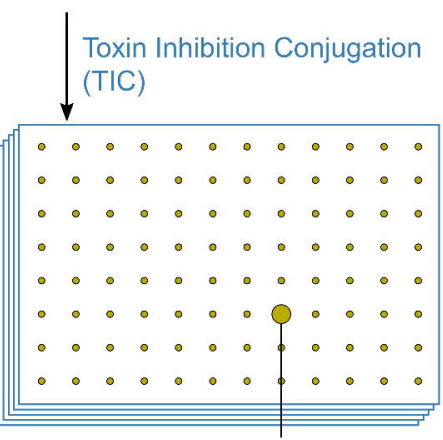

TA blocker
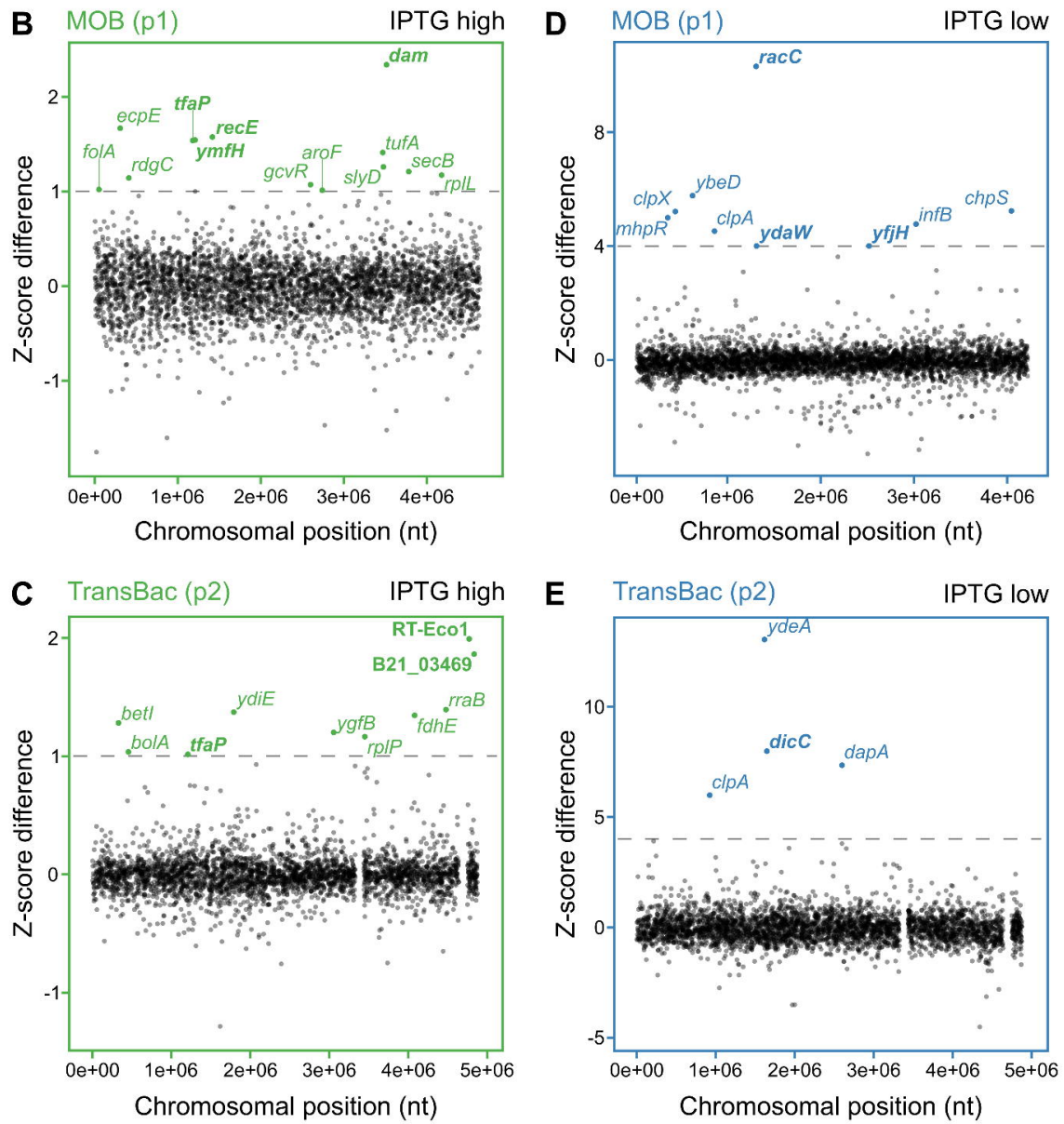
bioRxiv preprint doi: https://doi.org/10.1101/2020.06.22.160242; this version posted June 22, 2020. The copyright holder for this preprint (which was not certified by peer review) is the author/funcer ichphas 9 anted bioRxiv a license to display the preprint in perpetuity. It is

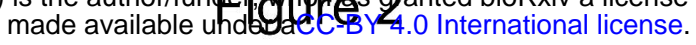

A

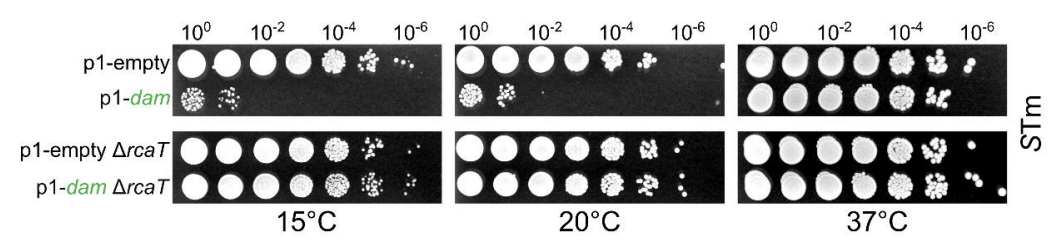

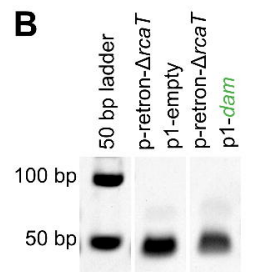

STm

C

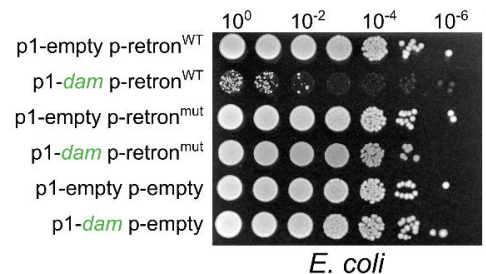

E. coli

D msDNA WT WT mut mut $\mathbf{E}$

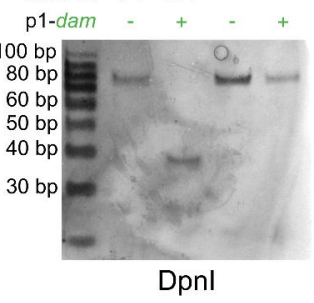

\section{E}

Inactive toxin

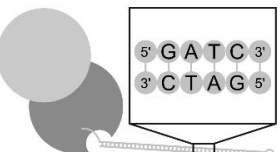

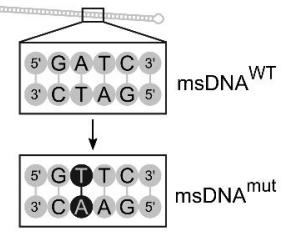

5. GATC 3. $\mathrm{msDNA}^{\mathrm{WT}}$

5. GTTC 3:

3. CAA G 5. $\mathrm{msNA}^{\text {mut }}$

Dpnl
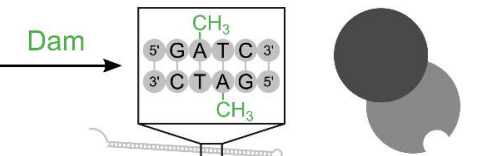
bioRxiv preprint doi: https://doi org/10.1101/2020.06.22.160242; this version posted June 22, 2020. The copyright holder for this preprint (which was not certified by peer review) is the author/funder, who has granted bioRxiv a license to display the preprint in perpetuity. It is made available under aCC-BY 4.0 International license.

\section{Figure 3}

A

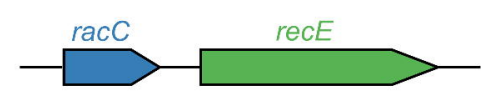

B

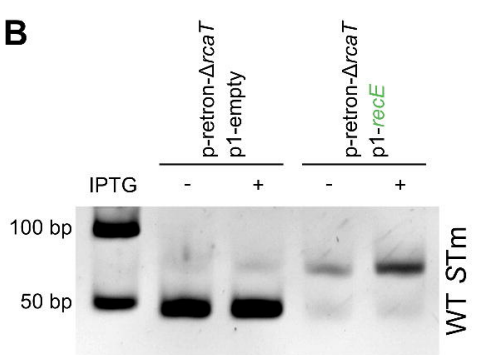

C

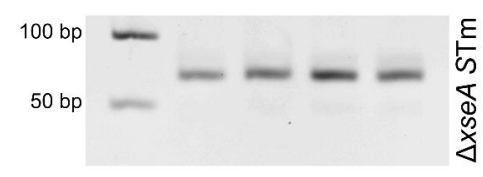

D

D Inactive toxin

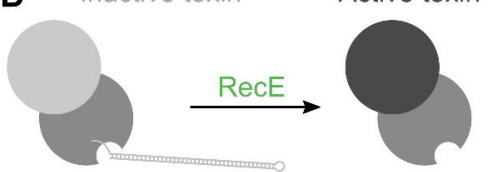

E

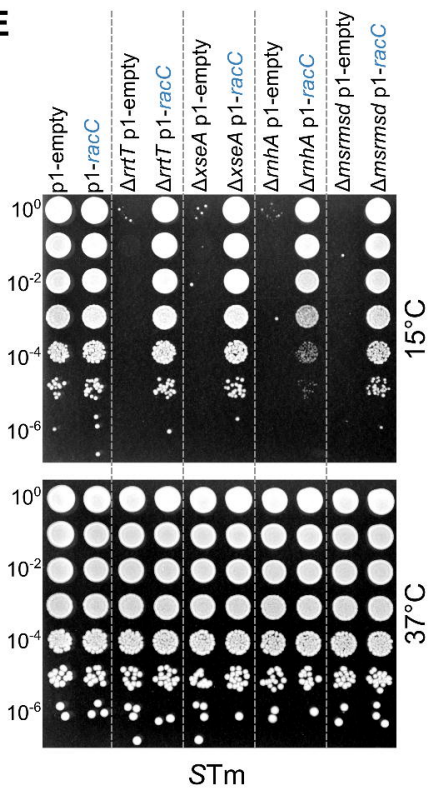

F Active toxin

Inactive toxin

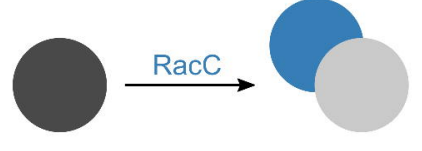


bioRxiv preprint doi: https://doi.org/10.1101/2020.06.22.160242; this version posted June 22, 2020. The copyright holder for this preprint (which was not certified by peer review) is the author/funde made available under 8 dd 4 \& International license.

$\mathbf{A}$
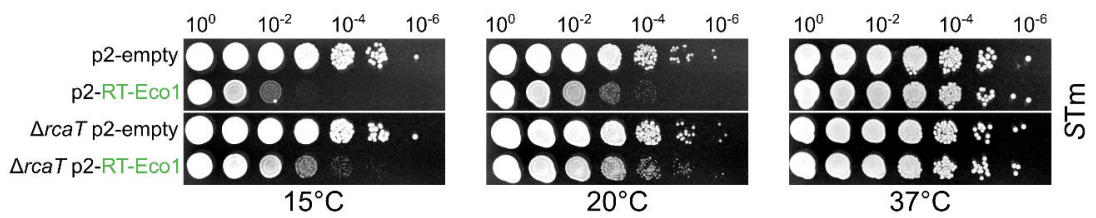

B

D

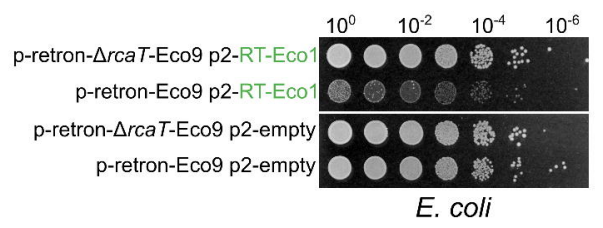

p1-msrmsd $\frac{\text { p-retron }}{-\quad \text { wT WT mut mut }} \frac{\text { p-retron- } \Delta r c a T}{-\quad \text { wT wT mut mut }}$ p2-RT-ECo1

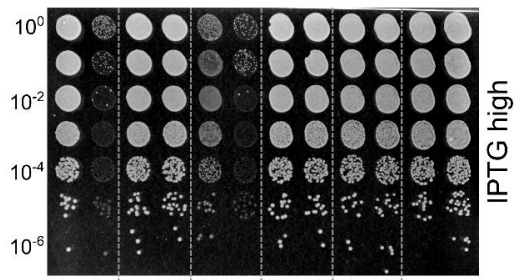

C
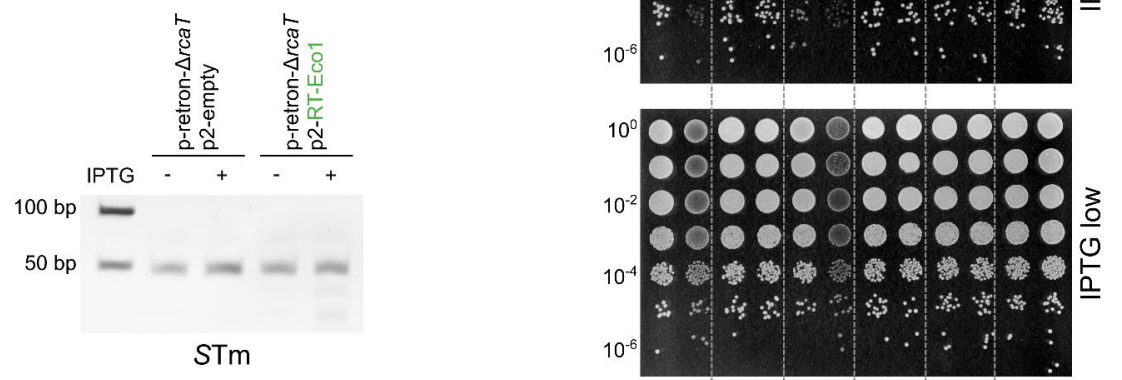

E
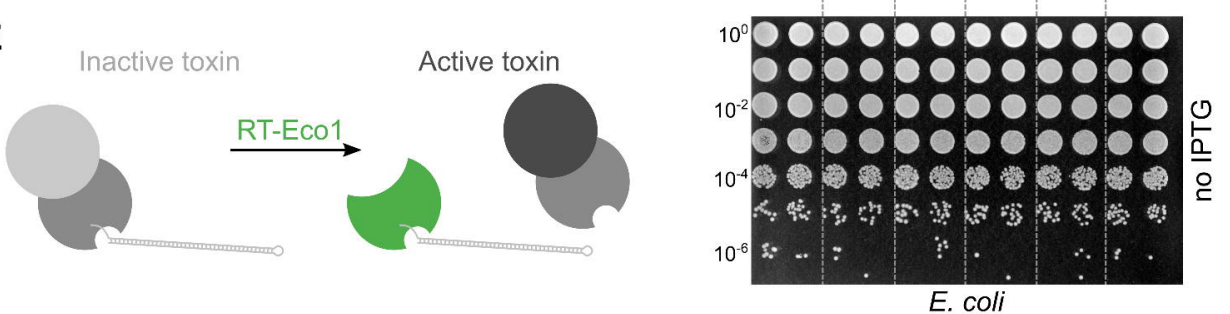
bioRxiv preprint doi: https://doi.org/10.1101/2020.06.22.160242; this version posted June 22, 2020. The copyright holder for this preprint (which was not certified by peer review) is the author/funder made available under 1 getik

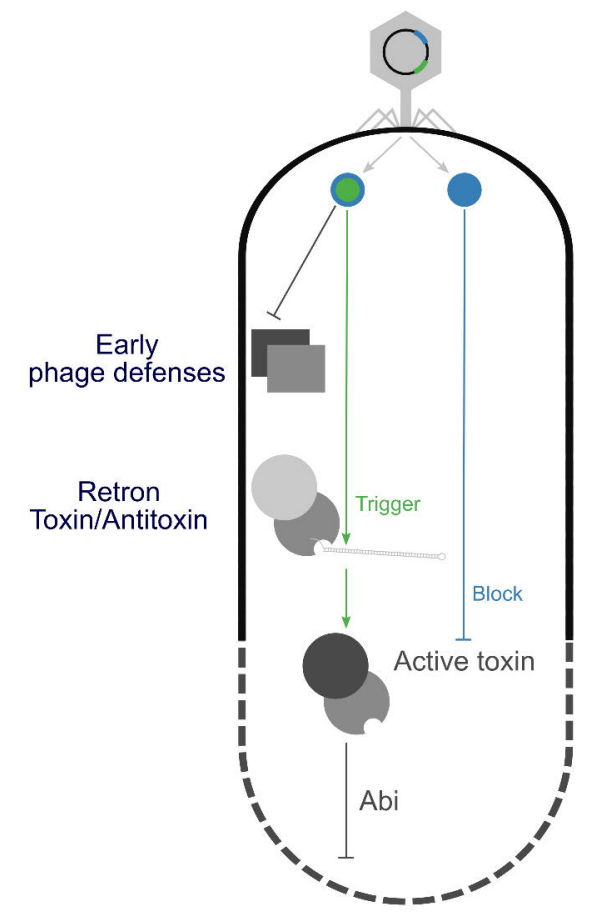


bioRxiv preprint doi: https://doi.org/10.1101/2020.06.22.160242; this version posted June 22, 2020. The copyright holder for this preprint (which was not certified by peer review) is the author/mpr. made availablentief alditionternational license.

A

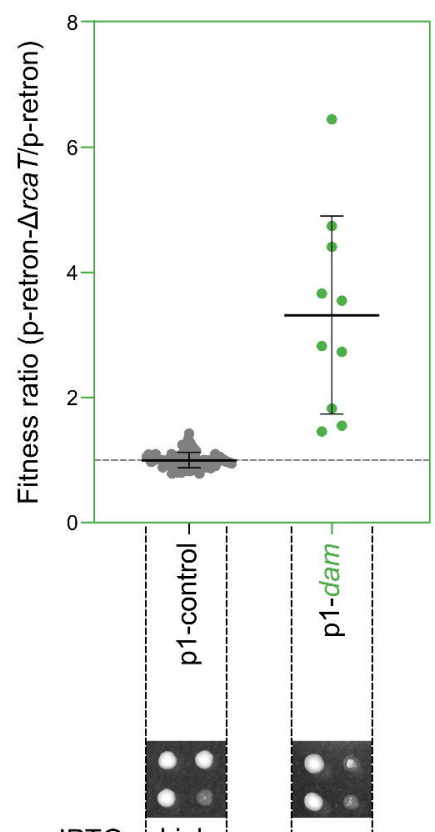

IPTG

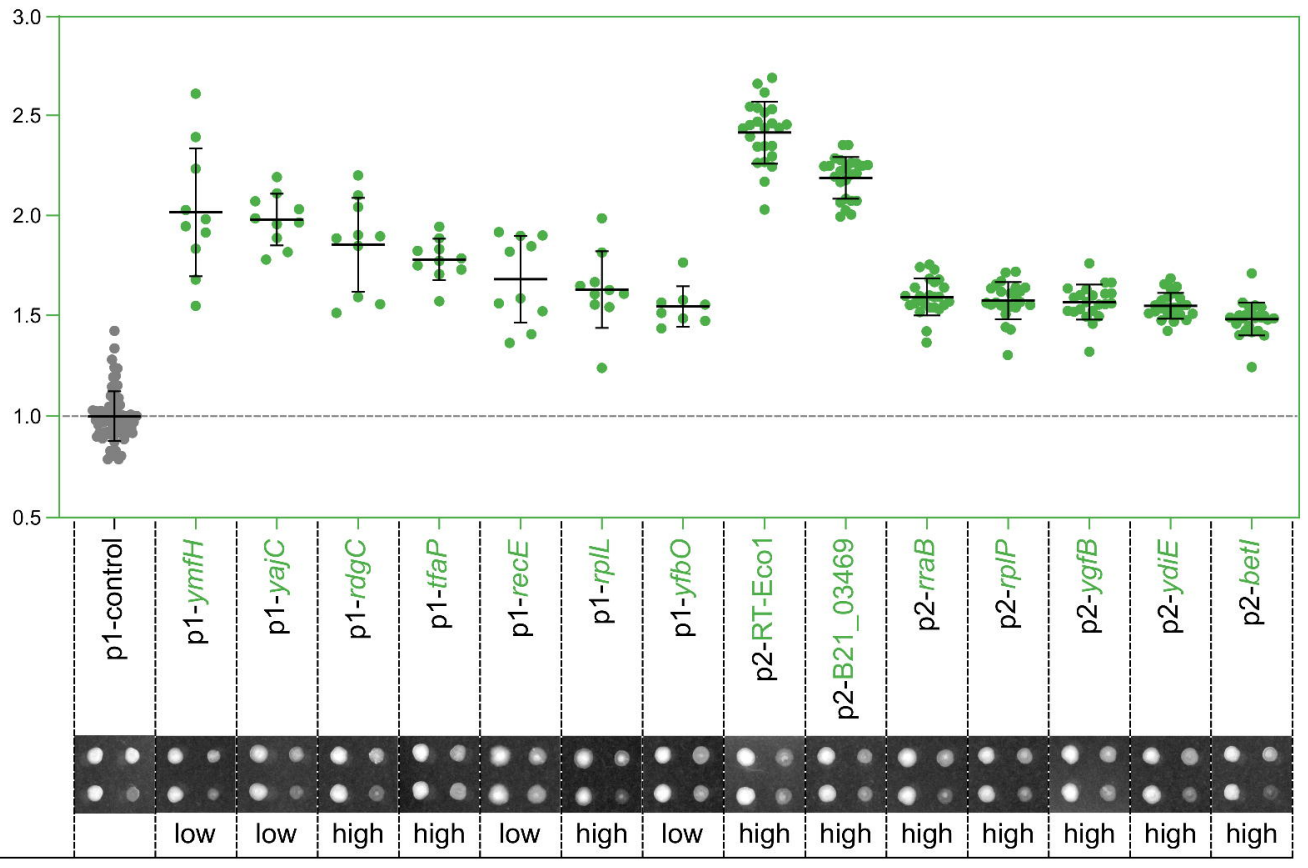

E. coli p-empty p-retron 100

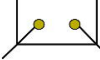
p-retron p-rcaT $(\Delta r c a T)$

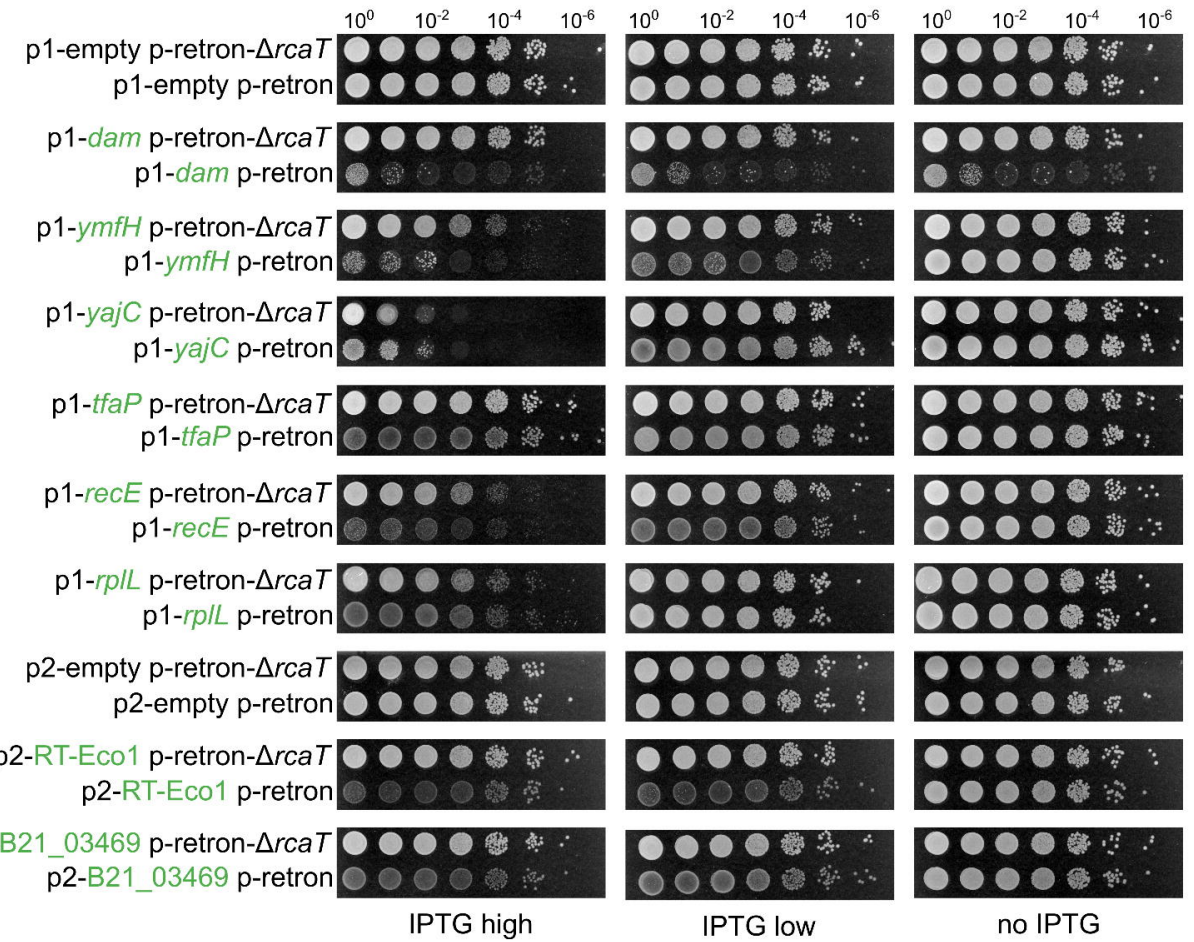


bioRxiv preprint doi: https://doi.org/10.1101/2020.06.22.160242; this version posted June 22, 2020. The copyright holder for this preprint (which was not certified by peer review) is the author/fund J made available urfeterace 4.6 te hational license.
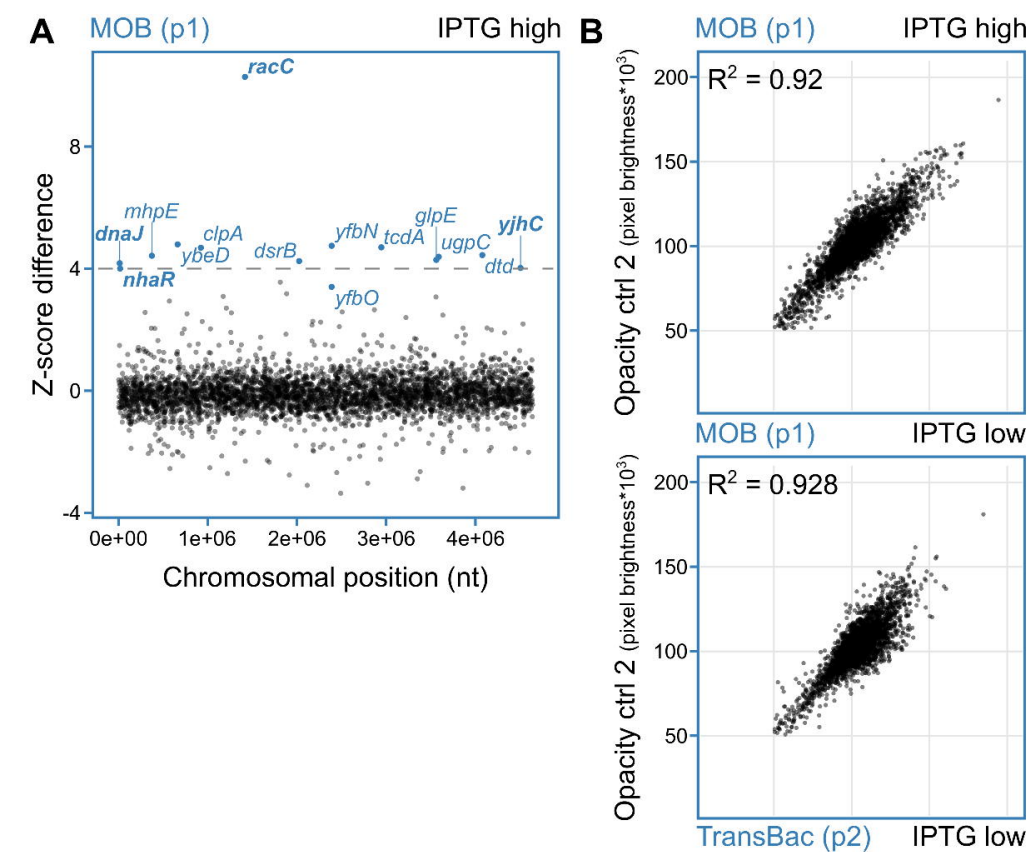

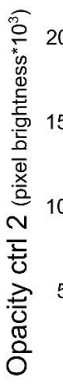

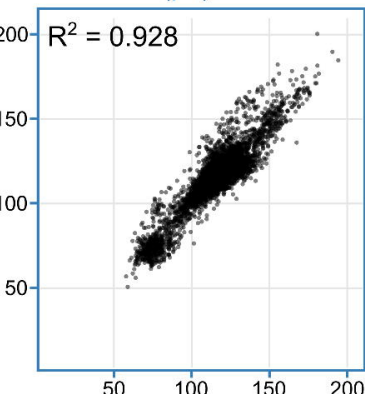

Opacity ctrl 1 (pixel brightness* ${ }^{*} 0^{3}$ )

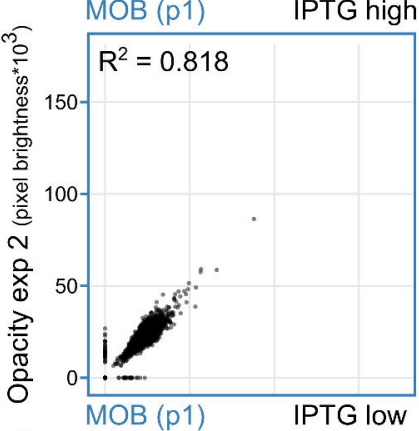

C

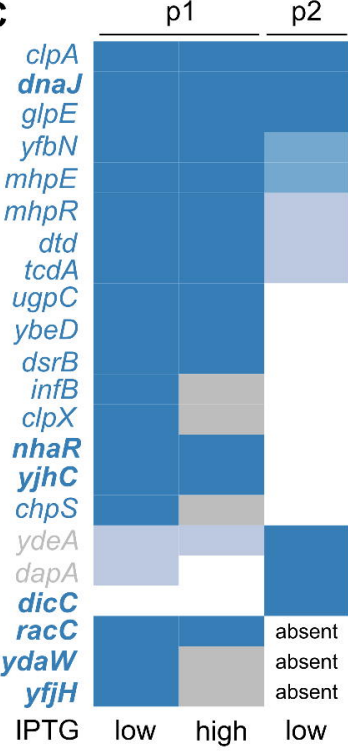

percentile

95-100 $90-95$ $85-90$ $0-80$ NA

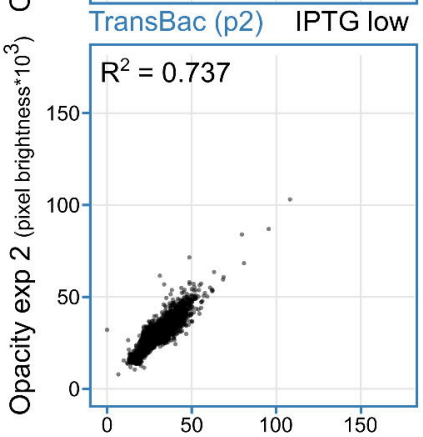

Opacity exp 1 (pixel brightness ${ }^{*} 10^{3}$ ) 
bioRxiv preprint doi: https://doi.org/10.1101/2020 06.22.160242; this version posted June 22, 2020. The copyright holder for this preprint (which was not certified by peer review) is the author/funder, who has granted bioRxiv a license to display the preprint in perpetuity. It is

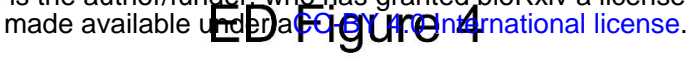
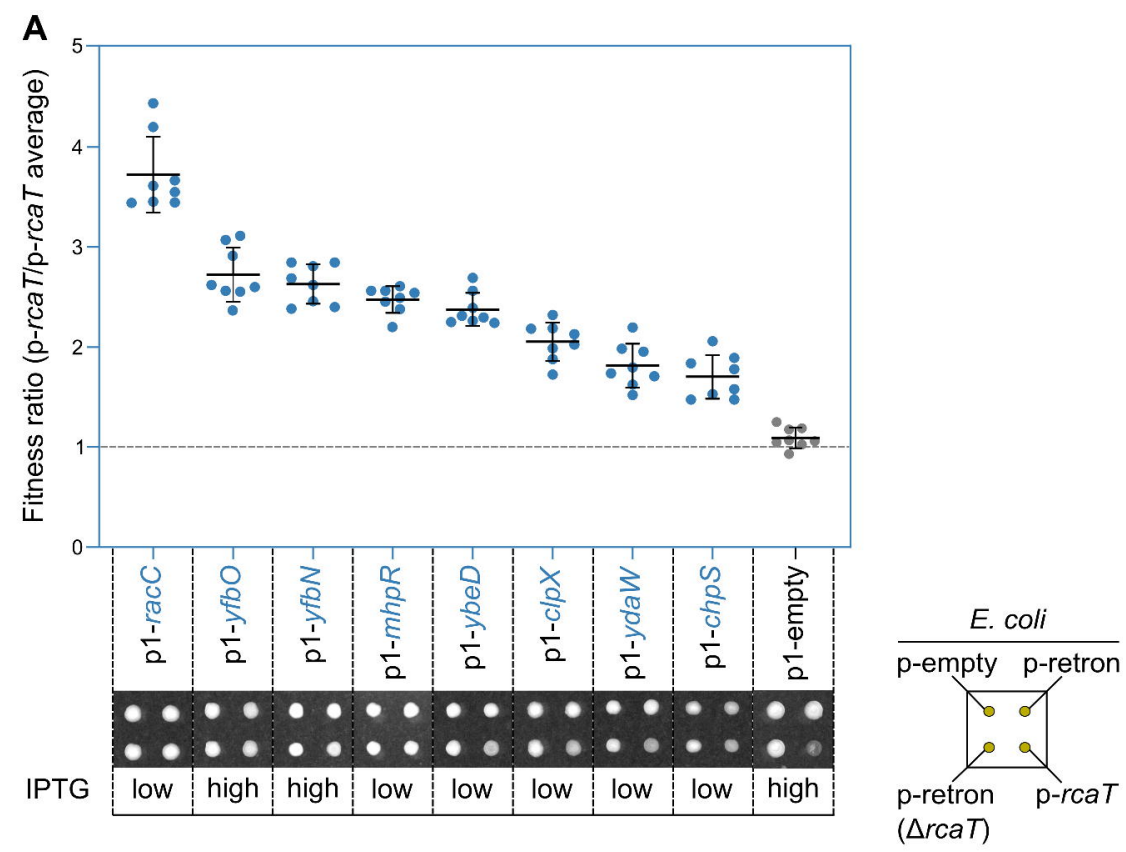

B

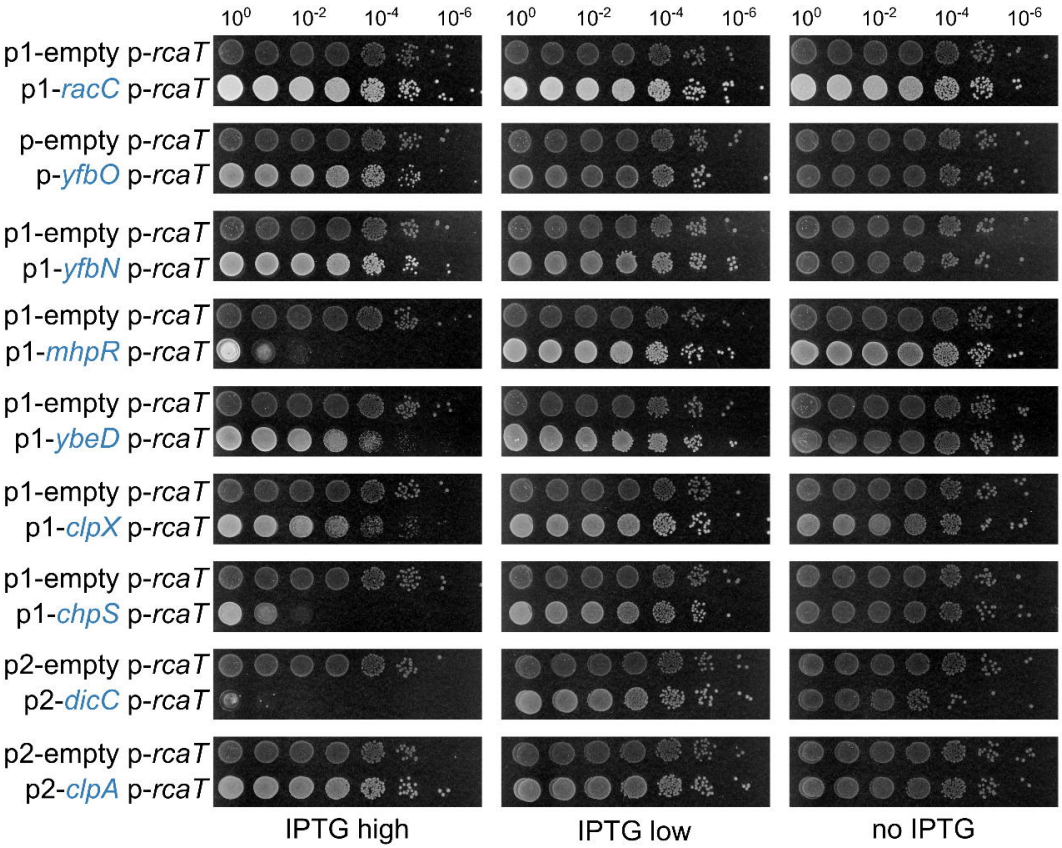


bioRxiv preprint doi: https://doi.org/10.1101/2020.06.22 160242; this version posted June 22, 2020. The copyright holder for this preprint (which was not certified by peer review) is the author/funder, who has granted bioRxiv a license to display the preprint in perpetuity. It is

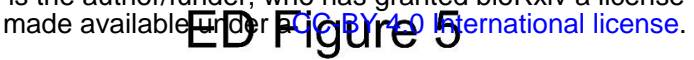
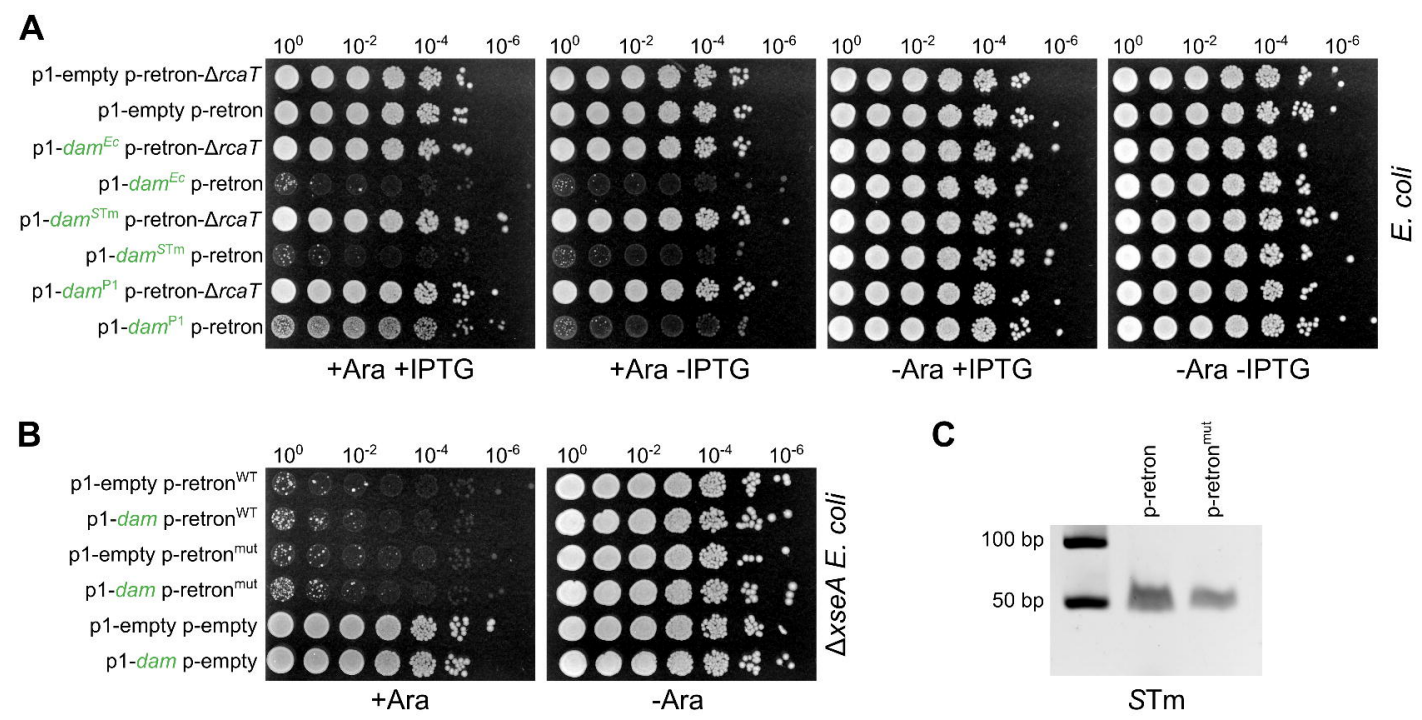
bioRxiv preprint doi: https://doi.org/10.1101/2020.06.22.160242; this version posted June 22, 2020. The copyright holder for this preprint (which was not certified by peer review) is the author/funder, who has granted bioRxiv a license to display the preprint in perpetuity. It is

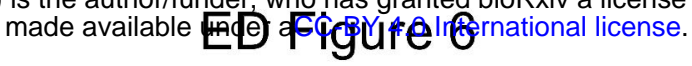

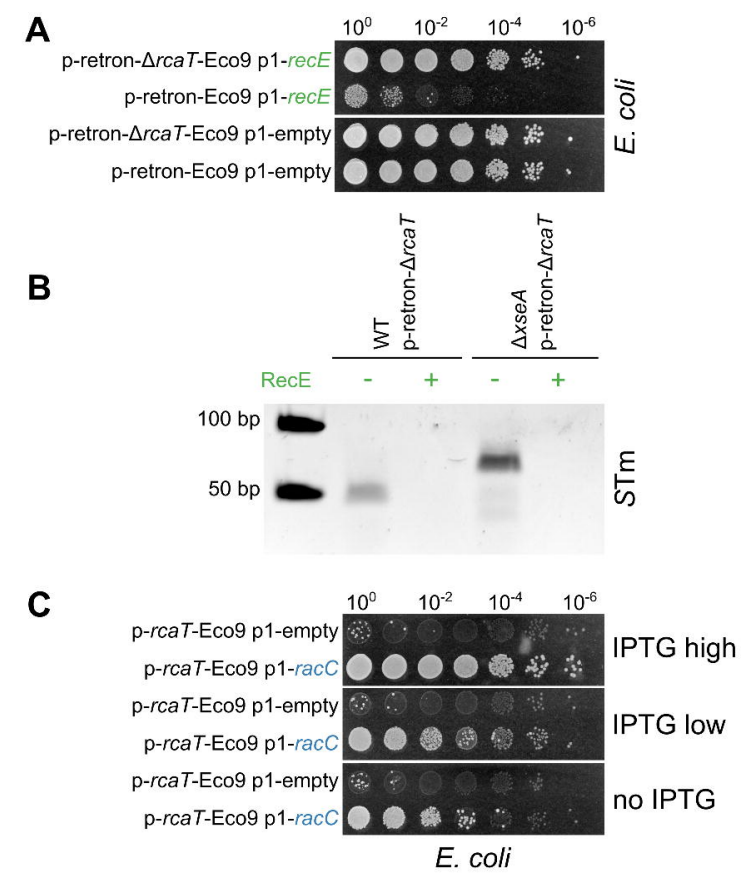


bioRxiv preprint doi: https://doi.org/10.1101/2020.06.22.160242; this version posted June 22, 2020. The copyright holder for this preprint (which was not certified by peer review) is the author/funder, who has granted bioRxiv a license to display the preprint in perpetuity. It is made available under aCC-BY 4.0 International license.

ED Figure 7

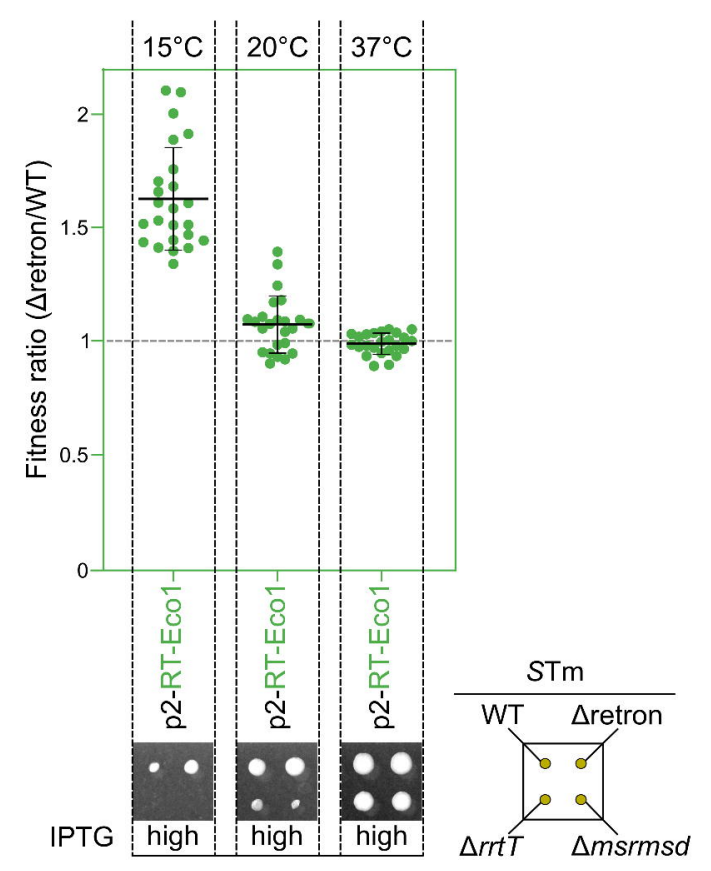

